OPEN ACCESS

Edited by:

Junbo Du,

Sichuan Agricultural University, China

Reviewed by:

Bijayalaxmi Mohanty,

National University of Singapore,

Singapore

Muthusamy Ramakrishnan,

Nanjing Forestry University, China

*Correspondence:

Fan Wei

wfmanuscript@163.com

Jianhua Miao

mih1962@vip.163.com

Specialty section:

This article was submitted to

Plant Abiotic Stress,

a section of the journal

Frontiers in Plant Science

Received: 05 November 2021

Accepted: 18 January 2022

Published: 10 February 2022

Citation:

Tang D, Quan C, Lin Y, Wei K,

Qin S, Liang Y, Wei F and Miao J (2022) Physio-Morphological,

Biochemical and Transcriptomic

Analyses Provide Insights Into Drought Stress Responses in Mesona chinensis Benth.

Front. Plant Sci. 13:809723. doi: 10.3389/fpls.2022.809723

\section{Physio-Morphological, Biochemical and Transcriptomic Analyses Provide Insights Into Drought Stress Responses in Mesona chinensis Benth}

\author{
Danfeng Tang 1,2, Changqian Quan 1,2, Yang Lin ${ }^{1,2}$, Kunhua Wei ${ }^{1,2}$, Shuangshuang Qin ${ }^{1,2}$, \\ Ying Liang ${ }^{1,2}$, Fan Wei ${ }^{1,2 *}$ and Jianhua Miao ${ }^{1,2 *}$
}

\footnotetext{
1 Guangxi Key Laboratory of Medicinal Resources Protection and Genetic Improvement, Guangxi Botanical Garden of Medicinal Plants, Nanning, China, ${ }^{2}$ Guangxi Engineering Research Center of TCM Resource Intelligent Creation, Guangxi Botanical Garden of Medicinal Plants, Nanning, China
}

Drought stress affects the normal growth and development of Mesona chinensis Benth (MCB), which is an important medicinal and edible plant in China. To investigate the physiological and molecular mechanisms of drought resistance in MCB, different concentrations of polyethylene glycol 6000 (PEG6000) (0, 5, 10 , and 15\%) were used to simulate drought conditions in this study. Results showed that the growth of MCB was significantly limited under drought stress conditions. Drought stress induced the increases in the contents of Chla, Chlb, Chla $+b$, soluble protein, soluble sugar, and soluble pectin and the activities of superoxide dismutase (SOD), catalase (CAT), total antioxidant capacity (TAC), hydrogen peroxide $\left(\mathrm{H}_{2} \mathrm{O}_{2}\right)$, and malondialdehyde (MDA). Transcriptome analysis revealed 3,494 differentially expressed genes (DEGs) (1,961 up-regulated and 1,533 down-regulated) between the control and 15\% PEG6000 treatments. These DEGs were identified to be involved in the 10 metabolic pathways, including "plant hormone signal transduction," "brassinosteroid biosynthesis," "plant-pathogen interaction," "MAPK signaling pathway-plant," "starch and sucrose metabolism," "pentose and glucuronate interconversions," "phenylpropanoid biosynthesis," "galactose metabolism," "monoterpenoid biosynthesis," and "ribosome." In addition, transcription factors (TFs) analysis showed 8 out of 204 TFs, TRINITY_DN3232_c0_g1 [ABA-responsive element (ABRE)-binding transcription factor1, AREB1], TRINITY_DN4161_c0_g1 (auxin response factor, ARF), TRINITY_DN3183_c0_g2 (abscisic acid-insensitive 5-like protein, ABI5), TRINITY_DN28414_c0_g2 (ethylene-responsive transcription factor ERF1b, ERF1b), TRINITY_DN9557_c0_g1 (phytochrome-interacting factor, PIF3), TRINITY_DN11435_c1_g1, TRINITY_DN2608_c0_g1, and TRINITY_DN6742_c0_g1, 
were closely related to the "plant hormone signal transduction" pathway. Taken together, it was inferred that these pathways and TFs might play important roles in response to drought stress in MCB. The current study provided important information for MCB drought resistance breeding in the future.

Keywords: Mesona chinensis Benth, biochemical analyses, transcriptome, plant hormone signal transduction, brassinosteroid biosynthesis, transcription factor

\section{INTRODUCTION}

Drought is a complex and natural phenomenon mainly caused by less precipitation in a certain period, which has the characteristics of frequency, intensity, and duration (Mishra and Singh, 2010). Drought stress is one of the most harmful abiotic stresses and can adversely affect the growth and development of crops (Fathi and Tari, 2016; Fahad et al., 2017; Hussain et al., 2018; Khan et al., 2020). In the agricultural production areas, especially in southern China, due to the changes of climate and environment and the development of the economy in recent decades, severer and more frequent drought periods seriously hinder plant growth and development resulting in a large number of agricultural production losses (Tang et al., 2019).

Water is one of the important environmental conditions for crop growth and development, which has a significant impact on crop morphology, physiological metabolism, yield, and quality. Drought stress affects the water relations of plants at the cellular, tissue, and organ levels causing physical damage, physiological and biochemical disruptions, and molecular changes (Beck et al., 2007) like photosynthesis, respiration, antioxidant, hormonal metabolism, and gene expression (Bhargava and Sawant, 2013). Under drought stress, the relative water content, chlorophyll content, and plant biomass decrease significantly in two wheat cultivars (Ma et al., 2012). Plants can reduce osmotic potential through the accumulation of osmotic substances (Bray, 1993), such as proline, soluble sugar, betaine, organic acid, free amino acid, and other organic substances (Wang et al., 2010) to maintain the normal water demand and promote drought tolerance. Drought stress can disturb the self-regulation balance of cells, lead to the excessive accumulation of reactive oxygen species (ROS), cause membrane peroxidation, changes in membrane fluidity and ion transport, damage of biological macromolecules, destruction of chloroplast structure, metabolic disorder, and finally result in plant death (Gill and Tuteja, 2010). In the process of long-term evolution, to maintain the steady-state balance of ROS in cells, plant cells have formed a complex antioxidant defense system, which includes superoxide dismutase (SOD), peroxidase (POD), catalase (CAT), ascorbic acid peroxidase (APX), and so on (Apel and Hirt, 2004; Jaleel et al., 2009). Plant hormones can be used as signal molecules to mediate the drought stress response of plants. Usually, plant hormones will react together to cope with drought stress, such as inhibiting the synthesis of growth and developmental hormones like auxin (IAA), gibberellin (GA), zeatin nucleoside (ZR), and promoting the synthesis of growth inhibitory hormones like abscisic acid (ABA) and jasmonic acid (JA) (Lu, 2020), etc. Among these, the plant hormone ABA plays a central role.
$\mathrm{ABA}$, as a long-distance signal, mediates the response of the whole plant to drought stress, and also as a cellular signal, regulates the expression of drought stress response genes (Zhang et al., 2006). Transcription factors (TFs) are the main regulators that control gene clusters. A single TF, called regulon, can regulate the expression of many target genes by the specific binding of the TF to cis-acting element in the promoters of the target genes; Dehydration-responsive element binding protein 1 (DREB1)/C-repeat binding factor (CBF) (DREB1/CBF) and DREB2 regulons function in ABA-independent gene expression, while the $A B A-r e s p o n s i v e ~ e l e m e n t ~(A B R E)$ binding protein (AREB)/ABRE binding factor (ABF) (AREB/ABF) regulon functions in ABA-dependent gene expression (Nakashima et al., 2009). In addition, TFs such as AP2/ERF, MYB, MYC, NAC, and $W R K Y$ are also involved in abiotic stress-responsive gene expression (Zhou et al., 2020).

Mesona chinensis Benth (MCB) is one of the important medicinal plants in tropical and subtropical areas, including southern China and Southeast Asian countries (Tang et al., 2020). MCB contains polysaccharides, flavonoids, triterpenoids, phenols, and other chemical components (Lin et al., 2013) with multiple biological activities, such as antioxidation (Yen and Hung, 2000), antihypertensive effects (Yeha et al., 2009), hypolipidemic effects (Li et al., 2010), antibiosis (Liu and Feng, 2008), etc. MCB can grow in slope, forest, ditch, and stream or dry sandy land and possesses strong environmental adaptability, however, it is not resistant to drought and cold (Zhao et al., 2011). If the water shortage is serious in summer and the temperature is too low in winter, the MCB plants will grow poorly or even die, resulting in yield reduction (Su et al., 2008). In recent years, due to high temperature and less rainfall in autumn and winter in Guangxi, moderate drought often occurs and lasts for about 3 months (Ren et al., 2017), which seriously affects the sustainable development of the MCB industry. Therefore, analysis of physiological, biochemical, and molecular responses of MCB seedlings under drought stress is of great significance for the study of drought resistance of MCB under the background of global climate change.

Polyethylene glycol (PEG) can hinder the plant conducting tissues and simulation of drought stress by PEG induces drought stress on the plants (Jiang et al., 1995). PEG molecules with a molecular weight greater than 3000 are hard to enter the cell wall space (Rubinstein and Turner, 1982) and are not apparently absorbed (Tarkow et al., 1996). PEG induces significant water stress in plants and is nontoxic (Emmerich and Hardegree, 1990). PEG6000 is mainly used to determine the drought stress-related information of plants (Turkan et al., 2005). Studies have reported that 
PEG6000 is used to simulate drought in many medicinal plants, such as Astragalus membranaceus (Liang et al., 2021), Sophora alopecuroides (Yan et al., 2020), Fagopyrum tataricum (Huang et al., 2021), Sophora moorcroftiana (Li et al., 2015), etc. However, there are fewer reports on the mechanism of drought stress in Mesona Chinensis Benth. In this study, PEG6000 was used to simulate drought in an experimental room. To study the mechanism of drought resistance, different PEG6000 concentrations $(0,5,10$, and 15\%, named as PEG0, PEG5, PEG10, and PEG15, respectively) were utilized and the morphological, physiological, biochemical, and molecular changes were characterized and determined. The current study was of great significance for the study of drought resistance of MCB and provided important information for the improvement of drought tolerance in this plant.

\section{MATERIALS AND METHODS}

\section{Plant Materials and Treatments}

The cutting seedlings of MCB were used in this study. The cuttings were planted in germination boxes using small white stones and treated individually with 5, 10, and 15\% PEG6000 (W/V) solutions. The different concentrations (5, 10, and 15\%) of PEG6000 were prepared in $0.5 \times$ Hoagland solution and the $0.5 \times$ Hoagland solution was used as control $(\mathrm{CK})$. In total, there were four treatments (CK, PEG5, PEG10, and PEG15) in this study, and each contained three replicates. All the materials were cultured under about 2400 LUX red light intensity and at $26 \pm 1^{\circ} \mathrm{C}$ for $16 / 8 \mathrm{~h}$ (light/dark) periods. The seedlings were cultured with tap water for 3 days to make the MCB plants adapt to the environment, then different levels of PEG6000 solutions were used for drought treatments. The different concentrations of PEG6000 solutions were changed every 2 days. After 8 days of drought stress, at least three plantlets were sampled for data collection of agronomic traits, meanwhile, the 3rd-5th apical leaves were harvested for biochemical determination and RNA sequencing. The samples were immediately frozen with liquid nitrogen and stored at $-80^{\circ} \mathrm{C}$ for further analysis.

\section{Biochemical Determinations}

Three plants were harvested for measurements of the fresh weight of the whole plant, root, and above-ground, plant height, and the stem diameter, then we took the average values. The Chla and Chlb concentration was measured by using the method of Gao (2000). The soluble sugar (SS) content was determined by the anthrone colorimetry method (Buysse and Merckx, 1993) and the soluble protein (SP) content was measured by Coomassie brilliant blue G-250 staining method (Bradford, 1976). The SOD activity was determined as described by Giannopolitis and Ries (1977). The POD activity was determined according to the reference (Hemeda and Klein, 1990). The CAT activity estimation was performed according to Goldblith and Proctor (1950). Malondialdehyde (MDA) content was estimated according to the method of Kramer et al. (1991). Hydrogen peroxide $\left(\mathrm{H}_{2} \mathrm{O}_{2}\right)$ content was detected as described by Velikova et al. (2000). All the data were collected with three replicates.

\section{RNA Extraction, Library Preparation, and Illumina Sequencing}

The 3rd-5th apical leaves of seedlings from the control (CK) and 15\% PEG6000 treatments were used for RNA isolation by RNA extraction Kit (TRIzol Reagent, Invitrogen, Carlsbad, CA, United States). Then the RNA integrity and quality were assessed. We used the Illumina TruSeq ${ }^{\text {TM }}$ RNA sample preparation Kit (San Diego, CA, United States) to prepare RNAseq transcriptome libraries. Then the RNAseq libraries were sequenced in a single lane on an Illumina Hiseq $\mathrm{x}$ ten/NovaSeq 6000 sequencer (Illumina, San Diego, CA, United States) for $2 \times 150$ bp paired-end reads.

\section{De novo Assembly and Annotation}

The raw paired-end reads were trimmed and quality controlled by Sickle $^{1}$ and SeqPrep ${ }^{2}$ with default parameters. Then the clean data were utilized to perform de novo assembly with Trinity $^{3}$ (Grabherr et al., 2011). All the assembled transcripts were searched against the NCBI protein non-redundant (NR), Cluster of Orthologous Groups of proteins (COG), and Kyoto Encyclopedia of Genes and Genomes (KEGG) databases using BLASTX to identify the proteins that had the highest sequence similarity with the given transcripts and a typical cut-off $E$-values $<1.0 \times 10^{-5}$ was set. BLAST2GO ${ }^{4}$ (Conesa et al., 2005) was employed to get Gene Ontology (GO) annotations of unique assembled transcripts for describing molecular functions (MF), biological processes (BP), and cellular components (CC). We used the KEGG (Goto and Kanehisa, 2000) to conduct metabolic pathway analysis. The RNA-seq data were deposited in Sequence Read Archive (SRA) database with accession number PRJNA777790.

\section{Differential Expression Analysis and Functional Enrichment}

To identify differential expression genes (DEGs) between the samples of control and 15\% PEG6000 (CK vs. 15\% PEG), we calculated the expression level of each transcript according to the transcripts per million reads (TPM) method and quantified gene abundances with RSEM (Li and Dewey, 2011). Differential expression analysis was performed using the DESeq2 (Love et al., 2014) with $Q$-value $\leq 0.05$, DEGs with $|\log 2 \mathrm{FC}|$ $>1$ and $Q$-value $\leq 0.05$ were considered as significantly differentially expressed genes. Moreover, functional-enrichment analyses including KEGG and GO were carried out to identify DEGs, which were significantly enriched in GO terms and metabolic pathways at Bonferroni-corrected $P$-value $\leq 0.05$ in comparison with the whole-transcriptome background. GO functional enrichment and KEGG pathway analysis were performed by Goatools and KOBAS (Xie et al., 2011).

\section{Quantitative Real-Time PCR Analysis}

We randomly selected 12 differentially expressed unigenes between PEG0 and PEG15 samples for qRT-PCR analysis. The

\footnotetext{
${ }^{1}$ https://github.com/najoshi/sickle

${ }^{2}$ https://github.com/jstjohn/SeqPrep

${ }^{3}$ http://trinityrnaseq.sourceforge.net/

${ }^{4}$ http://www.blast2go.com/b2ghome
} 
qRT-PCR primers were listed in Supplementary Table 1. The qRT-PCR was conducted by using ChamQ CYBR qPCR Master Mix (Vazyme, China) and QuantStudio 7 Flex system (Applied Biosystems, ABI, Waltham, MA, United States). The RNA and cDNA used in qRT-PCR were the same as those used for RNA sequencing. GAPDH was employed as an internal control and the $\mathrm{qPCR}$ system and condition were according to the reference (Tang et al., 2021). Three biological repeats were used for each.

\section{Statistics}

SPSS (Version.17, SPSS Inc., Chicago, IL, United States) software was used for one-way variance analysis (ANOVA). The data means were analyzed using the Duncan test for statistical significance $(P$-values $\leq 0.05)$.

\section{RESULTS}

\section{Drought Stress Strongly Limited the Growth and Development of Mesona chinensis Benth}

After 8 days of drought stress, the growth of MCB was significantly limited (Figure 1). With the increase of PEG6000 concentrations, the fresh weight of the whole plant, root, and above-ground significantly decreased (Figure 2). Compared to the control, the fresh weight of the whole plant, root, and above-ground was reduced by $36.19,19.00$, and $45.03 \%$ under 5, 10, and 15\% PEG6000 conditions, respectively. The contents of Chla, Chlb, Chla + b, soluble protein, soluble sugar, and soluble pectin were significantly increased with the increasing concentration of PEG6000 (Figure 3), which were positively correlated to PEG6000 concentration. Under 15\% PEG6000 condition, the contents of Chla, Chlb, Chla $+\mathrm{b}$, soluble protein, soluble sugar, and soluble pectin were increased by $103.95,65.26$, $88.80,608.16,209.47$, and $60.89 \%$, respectively, in comparison with the control.

\section{Drought Stress Promoted Antioxidant Enzyme Activities of Mesona chinensis Benth}

To better understand the possible mechanisms of the physiological response of MCB to drought stress, the activities of the antioxidant enzymes (SOD, POD, and CAT) and the total antioxidant capacity (TAC) were monitored under different concentrations of PEG6000. As shown in Figure 4, the SOD, POD, and CAT activities
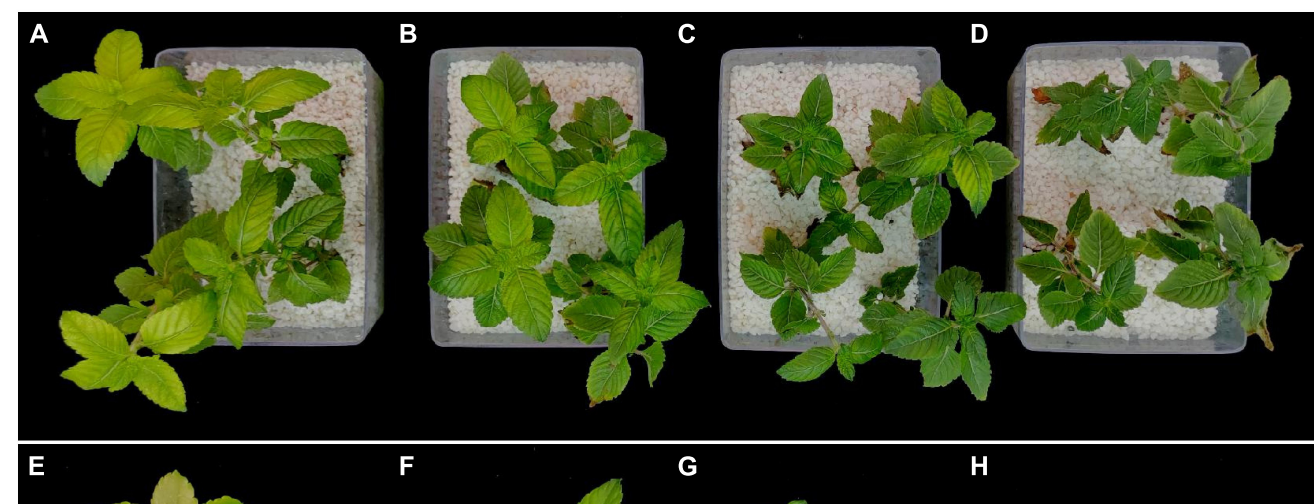

$\mathbf{G}$

H
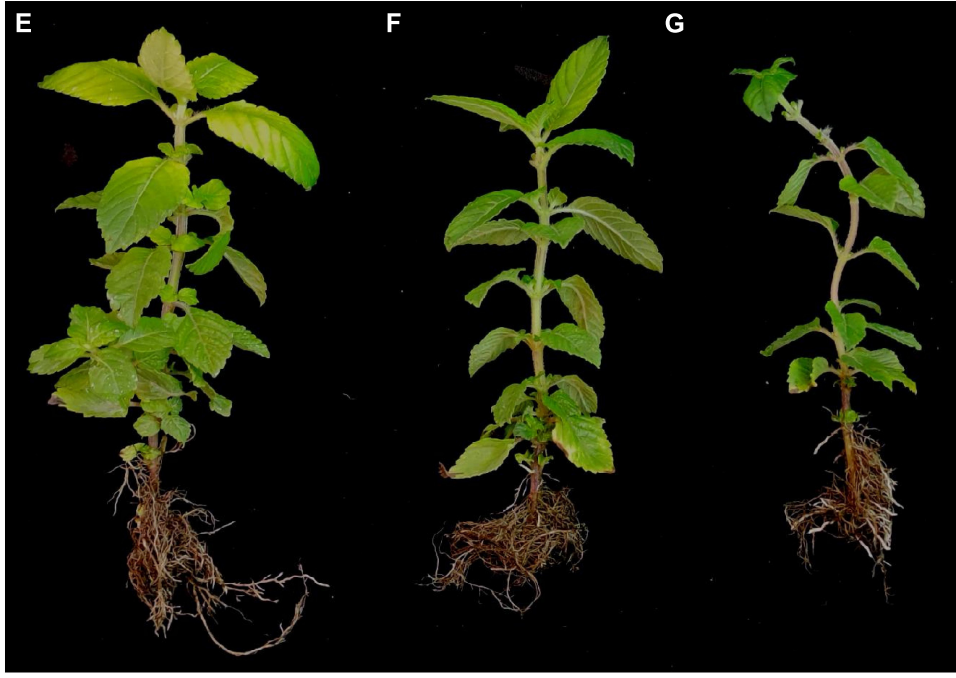

FIGURE 1 | Phenotypic changes of Mesona Chinensis Benth (MCB) under normal (CK) and drought stress conditions. (A,E) The plants under normal condition. (B,F) The plants under 5\% PEG6000 condition. (C,G) The plants under 10\% PEG6000 condition. (D,H) The plants under 15\% PEG6000 condition. 
- FW/plant $\mathbf{a W} /$ root $\mathbf{n}$ FW/aboveground (g)

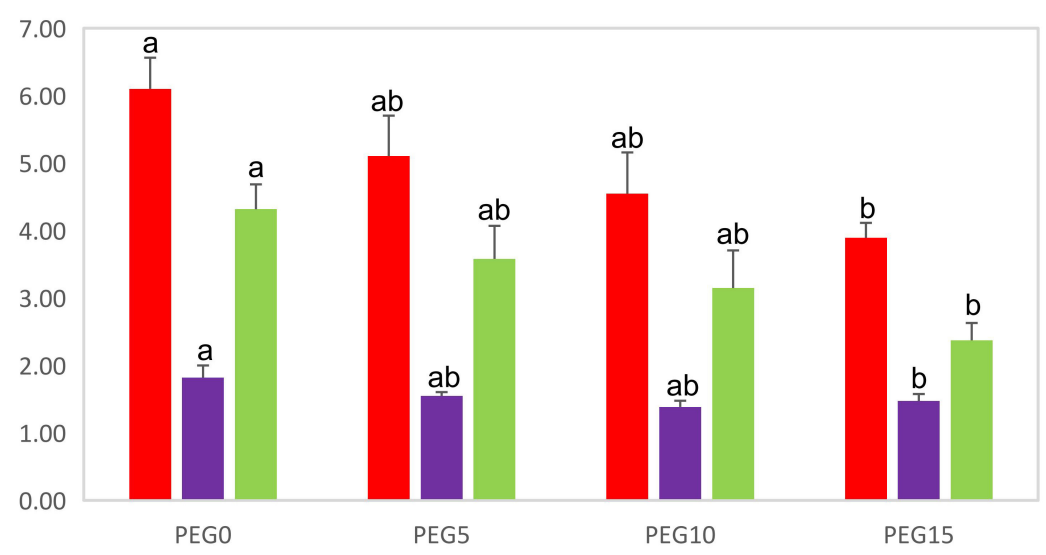

FIGURE 2 | Changes in the fresh weight of the whole plant, root, and above-ground under different drought stress conditions. Different letters indicate the significant difference at the 0.05 level.

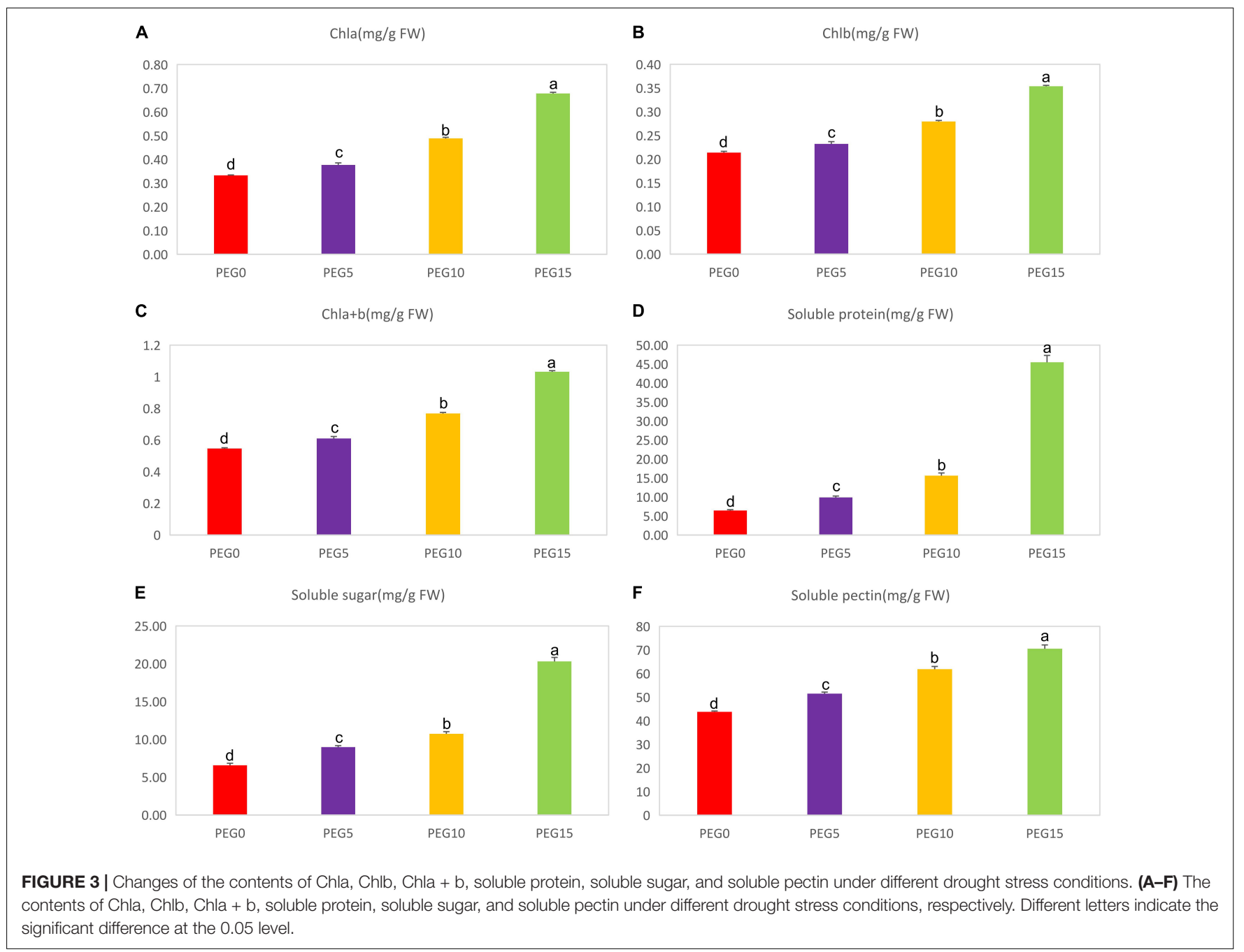




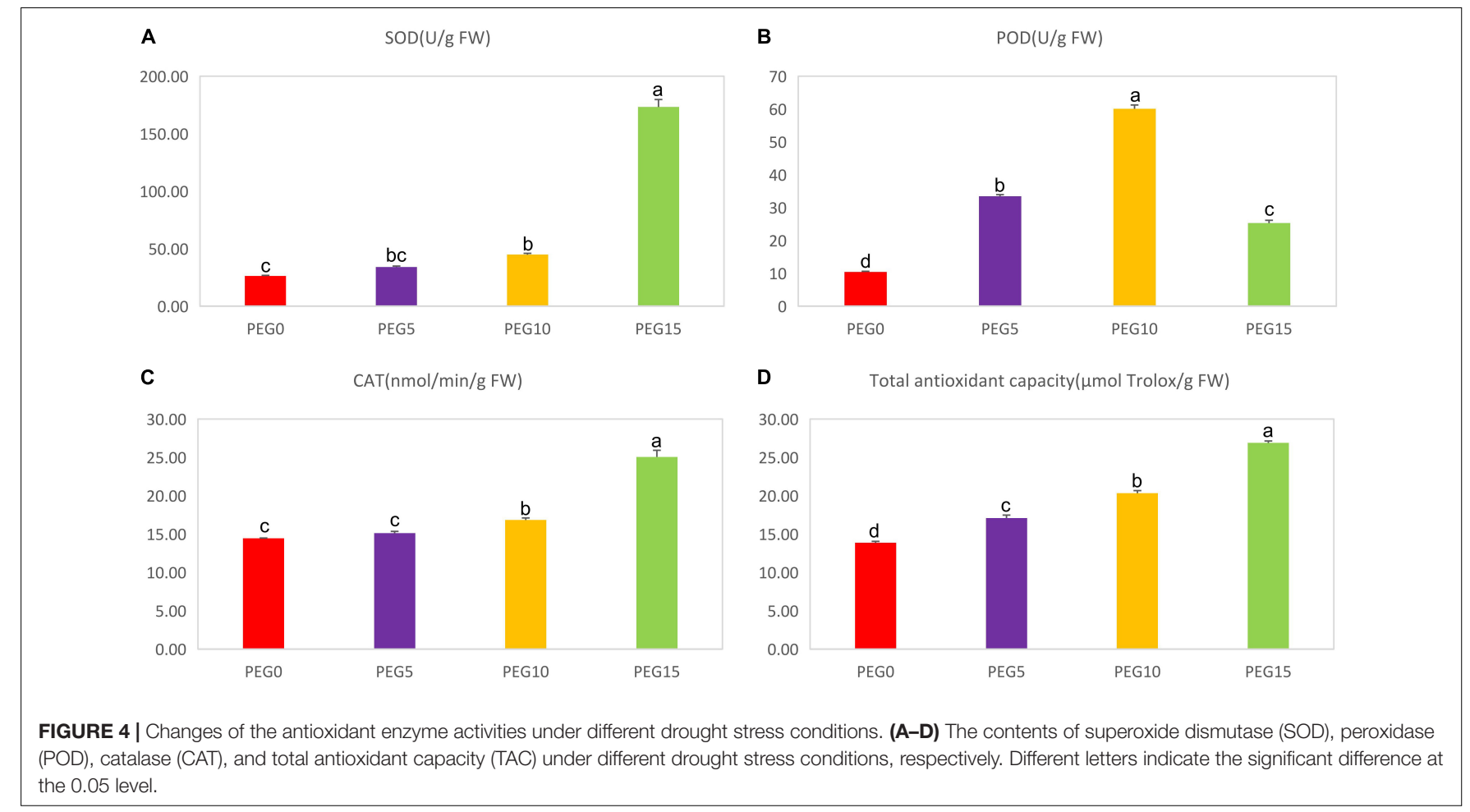

and the TAC were significantly affected in various drought treatments. The SOD, CAT, and TAC were increased with the increasing concentration of PEG6000. Under 15\% PEG6000 concentration, they were increased by 559.66, 73.56 , and $94.18 \%$, respectively, compared to the control. However, the POD activity was increased then decreased with the increasing concentration of PEG6000 (Figure 4B). Compared to the control, the POD activities in PEG5, PEG10, PEG15 treatments were increased by 220.32, 475.91, and $142.16 \%$, respectively.

\section{Drought Stress Improved Malondialdehyde and Hydrogen Peroxide Contents of Mesona chinensis Benth}

The MDA and $\mathrm{H}_{2} \mathrm{O}_{2}$ contents were also determined in various drought treatments. The contents of MDA and $\mathrm{H}_{2} \mathrm{O}_{2}$ were dramatically increased with the increase of PEG6000 concentrations (Figure 5). Under 5\% PEG6000 condition, they had no significant changes in comparison with the control. However, under 10 and 15\% PEG6000 conditions, the MDA content was increased by 35.67 and $100.22 \%$, and the $\mathrm{H}_{2} \mathrm{O}_{2}$ content was increased by 38.20 and $66.86 \%$, respectively.

\section{RNA Sequencing, de novo Assembly, and Functional Annotation}

To study the molecular mechanism of drought resistance in $\mathrm{MCB}$, we selected PEG0 and PEG15 treatments to conduct the transcriptome analysis of six samples in this study. A total of $40.69 \mathrm{~Gb}$ clean data were obtained and the clean data of each sample were more than 6.07 GB and the percentage of Q30 base was more than $95.6 \%$

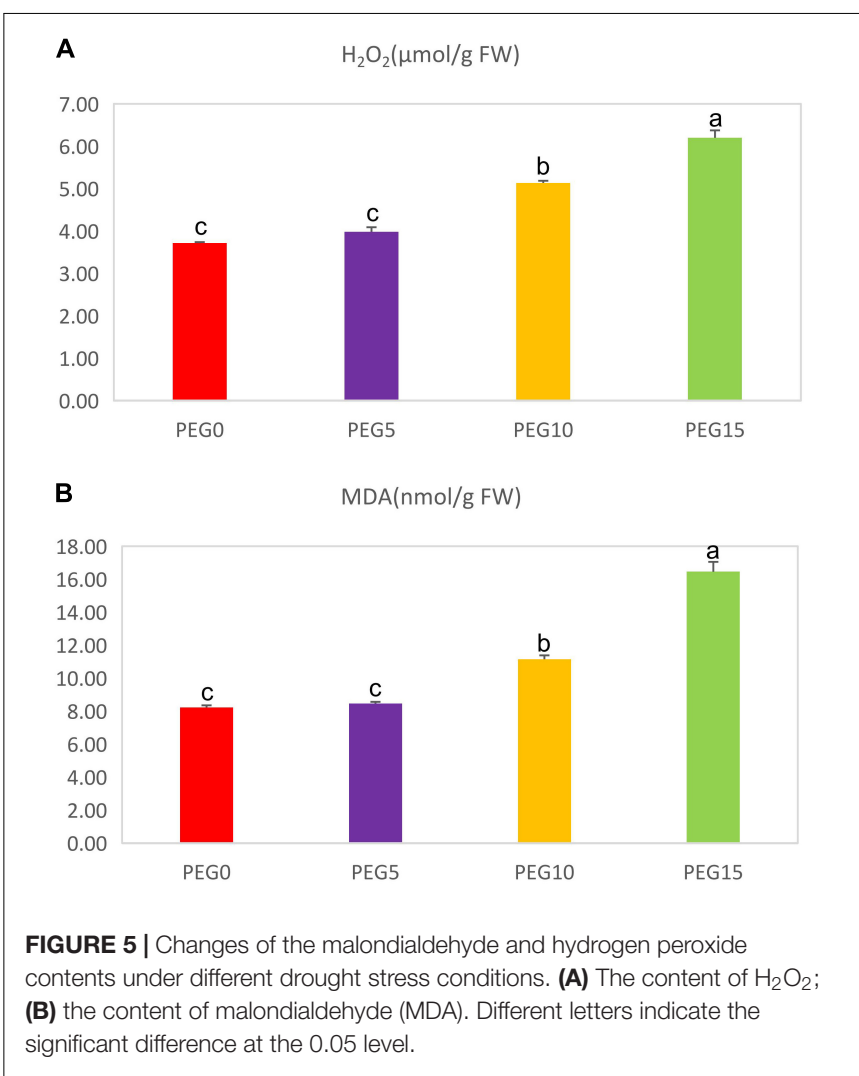




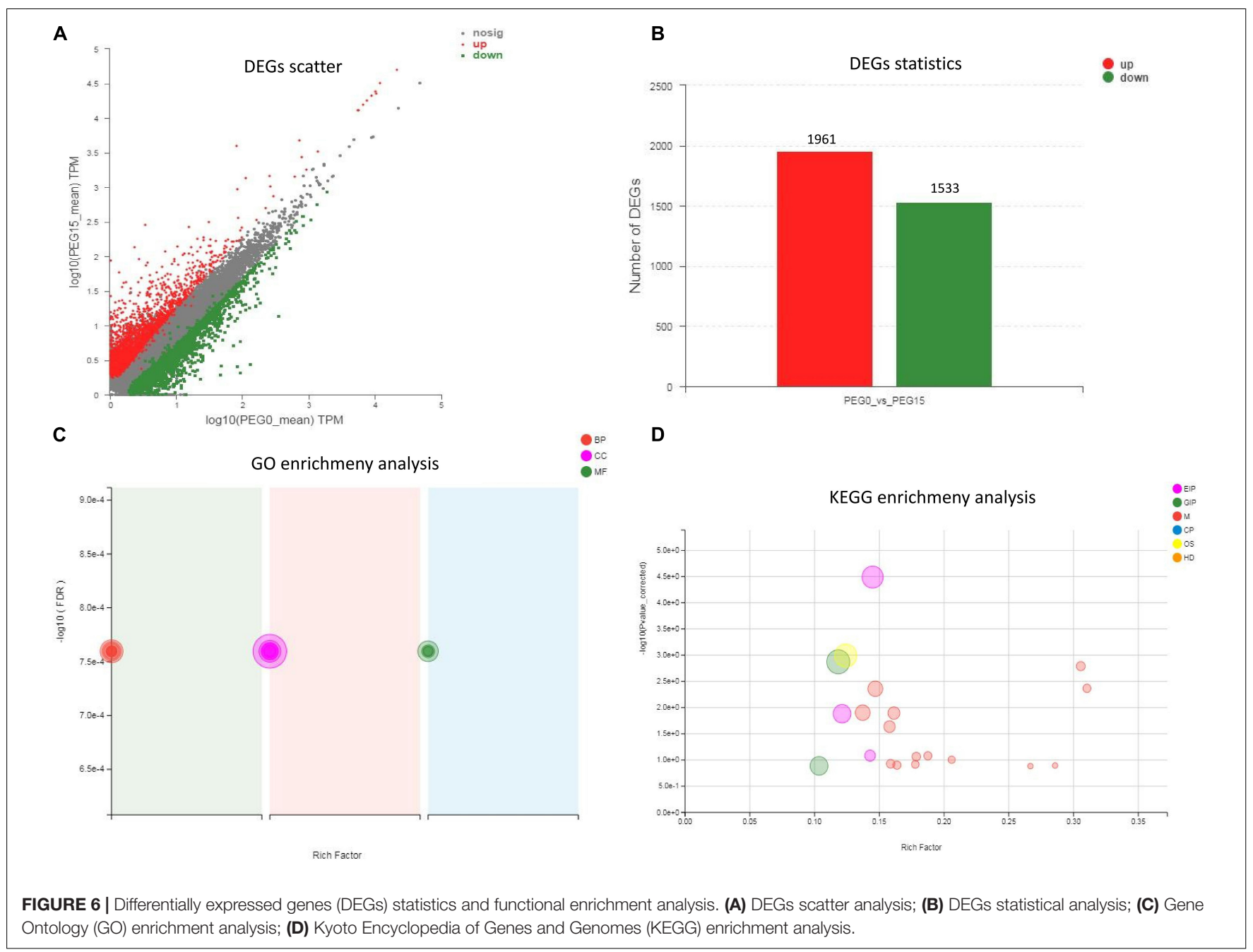

(Supplementary Table 2). All the clean data were de novo assembled by Trinity, and then the assembly results were optimized and filtered (TransRate ${ }^{5} ; \mathrm{CD}^{-\mathrm{HIT}^{6}}{ }^{\text {) }}$ and evaluated (BUSCO, Benchmarking Universal Single-Copy Orthologs ${ }^{7}$ ). We obtained 1,19,150 transcripts (total base, 12,38,02,946 bp; average length, $1039.05 \mathrm{bp}$; N50, 1,668 bp) and 71,785 unigenes (total base, 6,19,82,181 bp; average length, 863.44 bp; N50, $1,473 \mathrm{bp)}$ (Supplementary Table 3 ). All the transcripts were aligned against NR, Swiss-Prot, Pfam, COG, GO, and KEGG databases (Supplementary Figure 1). Cellular process and metabolic process were most enrichment in "biological process" (BP) and membrane part and cell part were the most in "cellular component" (CC), and binding and catalytic activity was significant enrichment in "molecular function" (MF) (Supplementary Figure 2). A total of 11,394 unigenes aligned with the top20 classifications and the pathways were divided into six categories including environmental information processing, metabolism, cellular processes, genetic

\footnotetext{
${ }^{5}$ http://hibberdlab.com/transrate/

${ }^{6}$ http://weizhongli-lab.org/cd-hit/

${ }^{7}$ http://busco.ezlab.org
}

information processing, organismal systems, and human diseases (Supplementary Figure 3).

\section{Identification of Differentially Expressed Genes}

According to the expression of unigenes/transcripts among different samples, analysis of expression distribution, venn, correlation, and PCA were performed (Supplementary Figure 4). Further, to study the drought resistance mechanism of MCB, the DEGs analysis between PEG0 and PEG15 was conducted. DESeq2 (Michael et al., 2014) software was employed for DEGs analysis, and the screening threshold was: | $\log 2 \mathrm{FC} \mid$ $>=1$ and $p$-adjust $<0.05$. A total of 3,494 DEGs were identified between the two treatments, including 1,961 up-regulated and 1,533 down-regulated unigenes (Figures 6A,B). GO enrichment showed the DEGs were involved in "lignin metabolic process," "metal ion transport," "pectin catabolic process," "cell wall," "oxidoreductase activity, acting on diphenols and related substances as donors, oxygen as acceptor," "galactosyltransferase activity," etc. (Figure 6C). KEGG enrichment revealed that the DEGs were associated with "plant hormone signal 
TABLE 1 | The metabolic pathways involved in response to drought stress in Mesona Chinensis Benth (MCB).

\begin{tabular}{lccc}
\hline Pathway id & Description & Gene num & P-value corrected \\
\hline map04075 & $\begin{array}{c}\text { Plant hormone signal } \\
\text { transduction }\end{array}$ & 63 & $3.23273 \mathrm{E}-05$ \\
map04626 & Plant-pathogen interaction & 70 & 0.001017808 \\
map03010 & Ribosome & 75 & 0.001335686 \\
map00905 & Brassinosteroid biosynthesis & 11 & 0.001618405 \\
map00902 & Monoterpenoid biosynthesis & 9 & 0.004288898 \\
map00940 & Phenylpropanoid biosynthesis & 31 & 0.004364363 \\
map00500 & Starch and sucrose metabolism & 31 & 0.01249589 \\
map00040 & Pentose and glucuronate & 20 & 0.012683802 \\
& interconversions & & 0.013092807 \\
map04016 & MAPK signaling pathway - & 44 & 0.023076204 \\
& plant & 18 &
\end{tabular}

transduction," "brassinosteroid biosynthesis," "plant-pathogen interaction," "starch and sucrose metabolism," "phenylpropanoid biosynthesis," and "pentose and glucuronate interconversions," etc. (Figure 6D and Table 1).

\section{Plant Hormone Signal Transduction in Response to Drought Stress}

Hormones play important roles in plant abiotic stress (Ryu and Cho, 2015). GO analysis of "plant hormone signal transduction" showed that the biological process and molecular function were the most enrichment categorizations (Figure 7A). Based on the KEGG analysis, we found that a total of 63 DEGs were enriched and showed close association with this pathway. Moreover, 16 DEGs were involved in "MAPK signaling pathway-plant" and 3 DEGs were involved in "circadian rhythm-plant" (Figure 7B). In the "plant hormone signal transduction" pathway, the 63 unigenes were mainly involved in the metabolism pathways of brassinosteroid (BR), ABA, ethylene (ETH), auxin (IAA), cytokinin (CTK), gibberellin (GA), and JA, including ethyleneresponsive TF ERF, ABA receptor PYR/PYL, Auxin-responsive protein SAUR, Jasmonoyl-L-amino acid synthetase JAR4, etc. (Figure 8A and Supplementary Table 4). In the "MAPK signaling pathway-plant" pathway, the 16 DEGs were associated with BAK1, ETR/ERS, ERF, MYC2, PYR/PYL, and PP2C (Figure 8B and Supplementary Table 5). In the "circadian rhythm-plant," 3 DEGs were related to PIF3 (Figure 8C and Supplementary Table 6). Therefore, it was inferred that various DEGs were involved in the "plant hormone and signal transduction" pathway in response to drought stress. In addition, among these DEGs, 33 unigenes were down-regulated and the remaining 30 unigenes were up-regulated under drought stress (Figure 9). These suggested that the expression of related genes in "plant hormone signal transduction," "MAPK signaling pathwayplant," and "circadian rhythm-plant" pathways might be closely related to drought response in MCB. In particular, ERF1 inhibited the production of MYC2 metabolites and MYC2 also inhibited the production of ERF1 metabolites in the "MAPK signaling pathway-plant" pathway (Figure 8B).

\section{Brassinosteroid Biosynthesis in Response to Drought Stress}

Brassinosteroids (BRs) regulate many physiological and developmental processes in plants, such as leaf expansion, cell elongation, flower development, light morphogenesis, stomatal development, and male sterility, and increase the plant tolerance to various kinds of abiotic stresses like drought, salinity, temperature, and heavy metal, etc. (Sharma et al., 2022). Combined with the results of the KEGG analysis of DEGs and "Plant hormone signal transduction," we found that brassinosteroid biosynthesis might play important roles in response to drought stress in MCB. Therefore, to further reveal that the brassinosteroid biosynthesis might be involved in drought stress response, the genes related to brassinosteroid biosynthesis were analyzed. As shown in Figure 10, a total of 11 DEGs were found in the "brassinosteroid biosynthesis" pathway and involved in multiple steps of brassinosteroid biosynthesis. The 11 DEGs were CYP90B1, CYP724B1, CYP92A6, CYP85A1, CYP734A1, and CYP90C1. Of these, 7 unigenes were downregulation, while 4 unigenes were up-regulation under drought stress conditions (Figure 11). It was indicated that these genes might be involved in drought stress.

\section{Differentially Expressed Transcription Factors Involved in Response to Drought Stress}

Transcription factors also play important roles in response to drought stress (Mun et al., 2017). In this study, a total of 1,294 and 2,667 TFs were identified in the unigenes and transcripts of $\mathrm{MCB}$, which were distributed in various $\mathrm{TF}$ families, such as $b H L H, C 2 C 2, A P 2 / E R F, N A C, b Z I P, W R K Y$, MYB_superfamily, etc. (Supplementary Table 7). In total, 204 TFs were identified in DEGs and distributed in 24 families, including bHLH, MYB_superfamily, C2C2, AP2/ERF, NAC, bZIP, $W R K Y$, and so on (Supplementary Table 8). GO enrichment analysis revealed that these 204 TFs were involved in "cellular developmental process," "positive regulation of macromolecule biosynthetic process," "cell differentiation," etc. in BP and "TF binding," "transcription regulatory region sequencespecific DNA binding," "transcription regulator activity," etc. in MF (Figure 12A). Further KEGG analysis unveiled that 8 TFs (TRINITY_DN3232_c0_g1, TRINITY_DN4161_ c0_g1, TRINITY_DN3183_c0_g2, TRINITY_DN28414_ c0_g2, TRINITY_DN11435_c1_g1, TRINITY_DN2608_c0_g1, TRINITY_DN6742_c0_g1, and TRINITY_DN9557_c0_g1) and two TFs (TRINITY_DN6742_c0_g1 and TRINITY DN9557_c0_g1) were associated with "plant hormone signal transduction" and "circadian rhythm-plant" pathways, respectively (Figure 12B and Supplementary Table 9). It was indicated that these TFs might be closely related to drought stress.

\section{qPCR Verification}

To verify the accuracy of transcriptome data, we selected 12 genes (eight protein-coding genes and four TFs) for $\mathrm{qPCR}$ analysis. The results showed that the fold changes of nine 
genes were in accordance with those of the transcriptome data. However, the fold changes of CYP90B1, CYP85A1, and CYP734A1 differed (Table 2).

\section{DISCUSSION}

\section{Drought Stress Significantly Limited the Growth of Mesona chinensis Benth}

Drought is a global problem that restricts the construction of the ecological environment and the distribution and productivity of plants. The effects of drought stress on plants are finally reflected in plant growth and development. Drought stress significantly affects the accumulation and distribution of plant biomass (Kashif, 2011). The drought adaptability of plants is closely related to the biomass distribution between shoot and root (Xu et al., 2015). Water stress significantly decreases the crop growth rate and fresh weight in tobacco (Shtereva et al., 2017). Similarly, in wheat, the plant root and shoot dry weight, plant height, leaf area, and yield are affected by drought (Hammad and Ali, 2014). In this study, drought stress significantly affected the fresh weight of the single plant, root, and above-ground parts of MCB (Figures 1, 2). Our results were consistent with the previous report (Yan et al., 2011).

The chlorophyll content is the most abundant pigment in the biosphere, which is directly related to plant photosynthesis, growth, and development and is an important indicator for the degree of stress (Kamble et al., 2015). Chlorophyll a (Chla) and chlorophyll b (Chlb) are the main components of chlorophyll. In the present study, the contents of Chla, Chlb, and Chla $+b$ were increased with the aggravation of drought stress (Figures $3 \mathbf{A}-\mathbf{C}$ ). It was indicated that the increase of chlorophyll content might help to protect the photosynthetic system of MCB to reduce the damage of drought stress.

Soluble sugar (SS) is an osmoregulation substance actively accumulated by plants under stress, which can not only reduce the osmotic potential of leaf cells but also improve the water absorption capacity of plants, so as to reduce drought stress damage (Yancey, 2001). Drought-induced or droughtinhibited soluble protein (SP) is usually employed to evaluate the drought tolerance of plants (Kang et al., 2005). Studies have demonstrated that the accumulation of SP and SS is

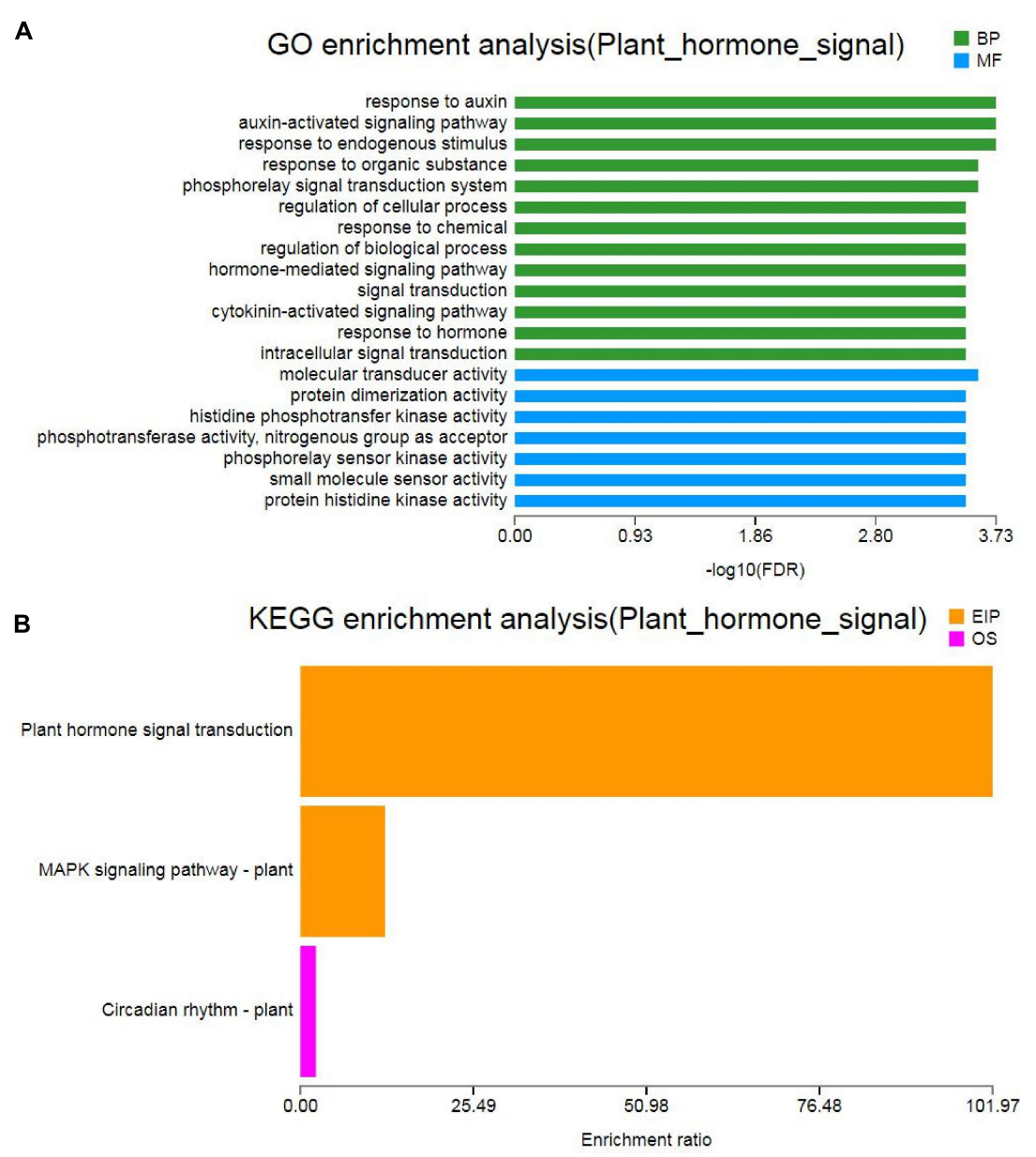

FIGURE 7| Gene Ontology and KEGG analysis of the "plant hormone signal transduction" pathway. (A) GO analysis of the "plant hormone signal transduction" pathway. (B) KEGG analysis of the "plant hormone signal transduction" pathway. 


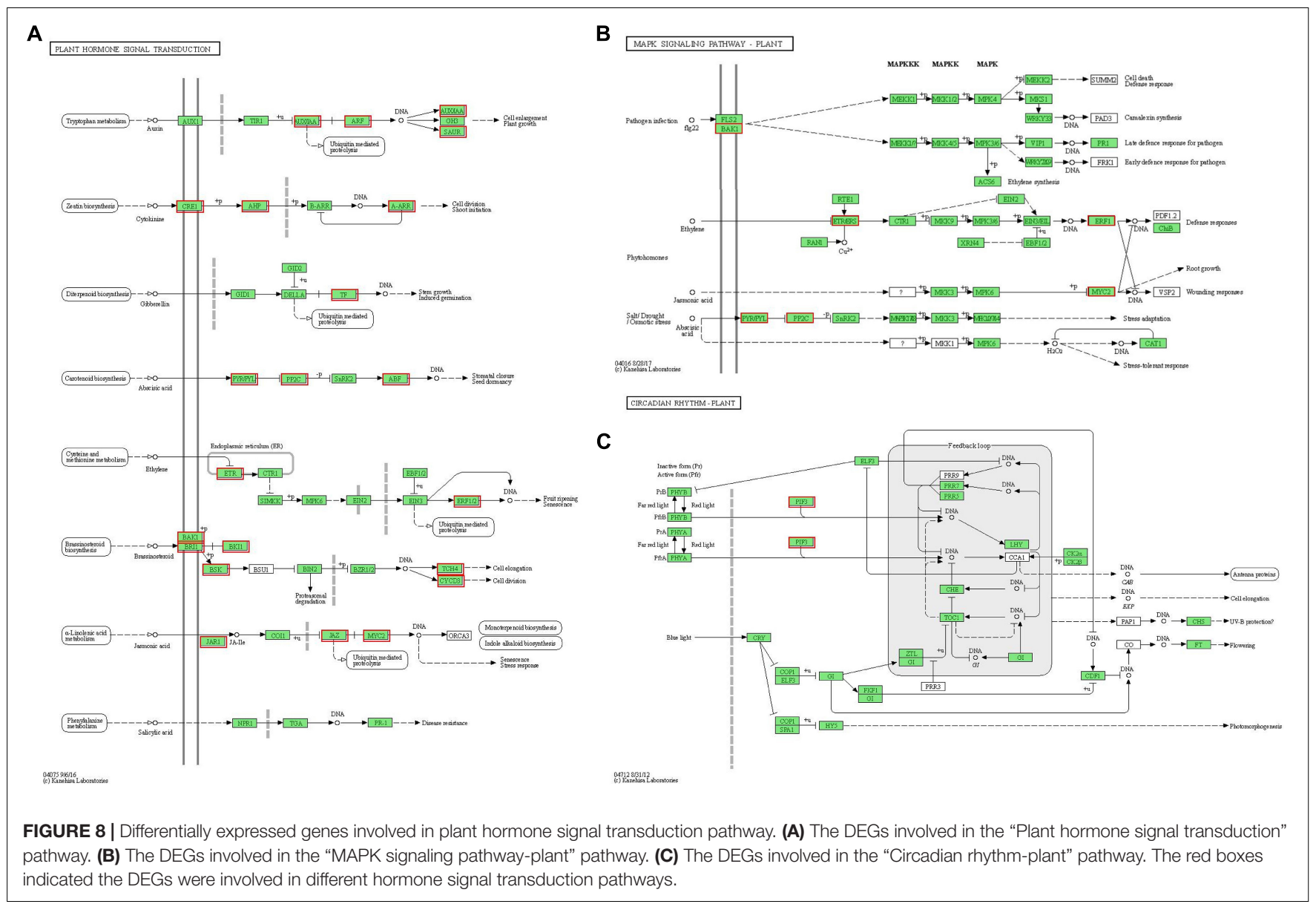

one of the most important responses of plants to drought stress (Silva et al., 2010; Li et al., 2014). In our investigation, the SS and SP contents were dramatically accumulated with the increasing PEG concentrations (Figures 3D,E). It was in line with the previous findings ( $\mathrm{Hu}$ et al., 2015). In addition, the soluble pectin content was also increased and had a positive correlation with drought stress (Figure 3F). Soluble pectin is an important index to measure the quality of $\mathrm{MCB}$, therefore it might also be used as a drought resistance indicator of MCB.

\section{Drought Stress Induced Anti-oxidative Defense and Osmotic Adjustments}

Drought stress interrupts the balance of the ROS (e.g., superoxide and $\mathrm{H}_{2} \mathrm{O}_{2}$ ) production and antioxidant defense mechanism in plants (He et al., 2017), and an excessive formation of ROS can result in oxidative damage in plants (Sharma et al., 2012). Multiple antioxidant enzymes, e.g., SOD, POD, and CAT, are associated with the response of a plant to drought stress, and the production of the antioxidant enzymes is considered as a protective mechanism of plants against drought stress ( $\mathrm{Li}$ and $\mathrm{Yi}, 2012$ ). SOD is the key enzyme in the active oxygen scavenger system because it catalyzes the dismutation of superoxide free radicals into
$\mathrm{H}_{2} \mathrm{O}_{2}$ and $\mathrm{O}_{2}$, and POD and CAT further convert $\mathrm{H}_{2} \mathrm{O}_{2}$ into $\mathrm{H}_{2} \mathrm{O}$ and $\mathrm{O}_{2}$, and then the damage caused by ROS is eliminated from plants (Wu et al., 2012; Hu et al., 2015). Total antioxidant capacity (TAC) reflects an integration activity of several antioxidants and is an important parameter in the analysis of biological matrices (Jaberiea et al., 2020). Here, the activities of SOD, CAT, and TAC were significantly increased with the aggravation of drought stress, while the POD activity was increased in PEG5 and PEG10, then decreased in PEG15 (Figure 4). It was inferred that POD, SOD, and CAT could effectively scavenge free radicals to reduce drought damage to MCB plants.

Malondialdehyde (MDA), a product of lipid peroxidation and $\mathrm{H}_{2} \mathrm{O}_{2}$, is generally used as a stress biomarker for the detection of membrane lipid peroxidation in plants (Yonny et al., 2017). The content of MDA is positively correlated with the damage degree of the plant plasma membrane (Zhong et al., 2021). Previous studies have reported an increased amount of $\mathrm{MDA}$ and $\mathrm{H}_{2} \mathrm{O}_{2}$ in response to drought stress (Cao et al., 2017). Drought stress induces a larger production of MDA in the leaves of tobacco (Khan et al., 2019). In our study, the MDA and $\mathrm{H}_{2} \mathrm{O}_{2}$ contents were increased with the increase of PEG6000 concentrations (Figure 5). These findings were supported by Yang et al. (2015), indicating that the plant plasma membrane was damaged to a certain 


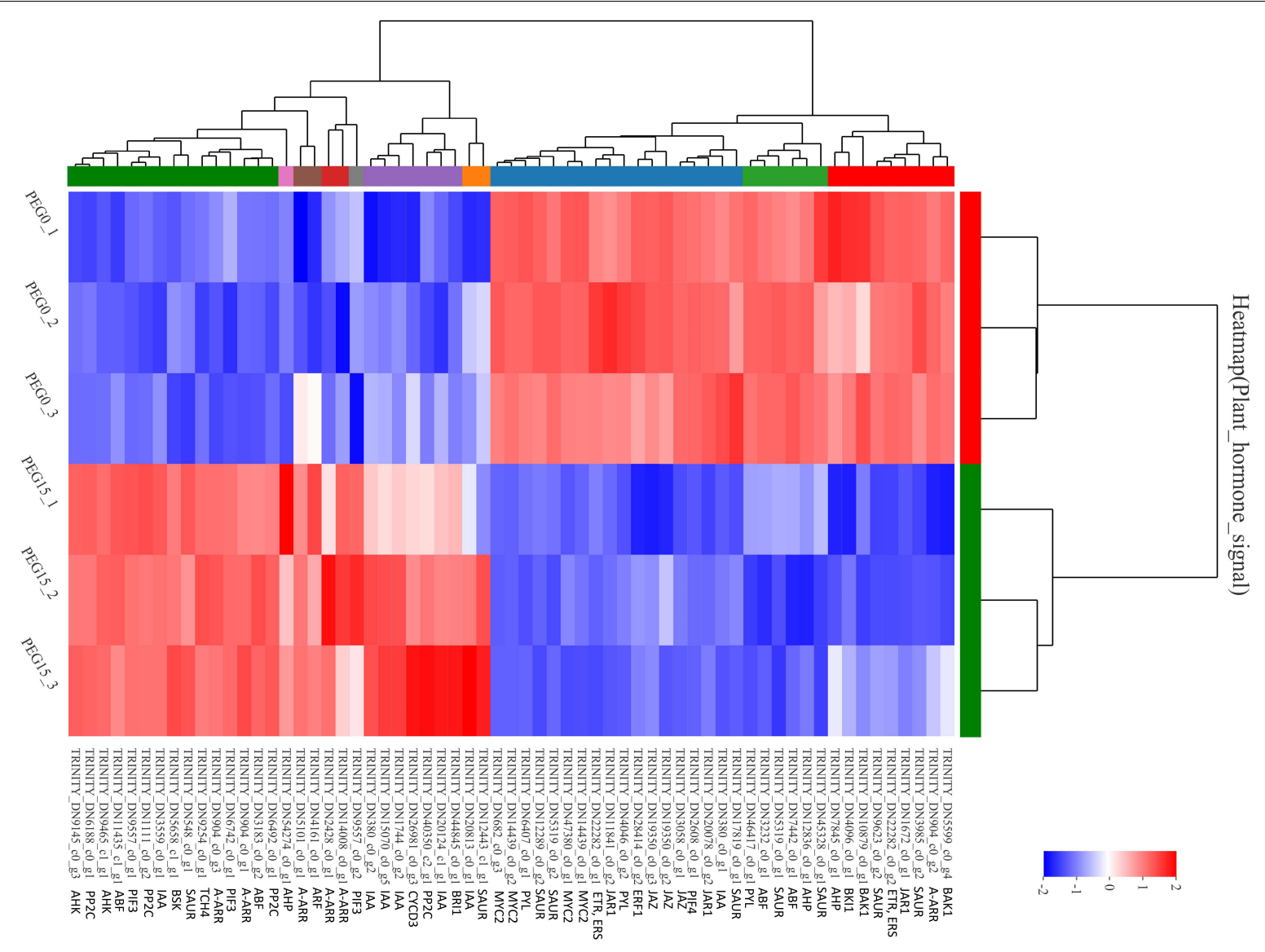

FIGURE 9 | The heat map of the DEGs involved in the "plant hormone signal transduction" pathway.

extent and the lipid peroxidation of the plasma membrane was gradually enhanced.

\section{Plant Hormone Signal Transduction Involved in Response to Drought Stress}

Phytohormone may be the crucial regulator in response to the harmful effects of various stress types and the coordination between the different hormone signaling is flexible in stress types and intensity and plant types under stress (Suzuki, 2016). $\mathrm{ABA}$ is an important plant hormone known to mediate the responses to drought stress, which results in the expression of genes related to the specific condition by initiating the cell signaling pathways (Lee and Luan, 2012). In the ABA signaling pathway, PYR/PYL/RCAR is the receptor, PP2C acts as a negative regulator, while SnRK2 acts as a positive regulator (Zhang et al., 2006; Khan et al., 2019). Under normal growth conditions, the contents of $\mathrm{ABA}$ and PYR/PYL/RCAR protein in plants are at a low level, and PP2C phosphatase binds to SnRK2 kinase and inhibits its activity, and the ABA signal remains silent; Whereas, the pathway is triggered by the binding of $\mathrm{ABA}$ to the PYR/PYL/RCAR receptor, which then sequesters the PP2C, allowing the downstream SnRK2s to activate or inactivate the targets by phosphorylation, which include KAT1, SLAC1, the
NADPH oxidase AtrbohF, and several $b$-ZIPs (Joshi-Saha et al., 2011). In this study, drought stress induced the differential expression of $P Y L, P P 2 C$, and $A B F$. In this pathway, 3 PYL genes were down-regulation, while $4 \mathrm{PP} 2 \mathrm{C}$ genes were up-regulation, and $4 \mathrm{ABF}$ genes (2 down-regulation and 2 up-regulation) were identified. These suggested that the down-regulation of $P Y L$ might enhance the up-regulation of $P P 2 C$, activate $S n R K 2 s$, promote the expression of $A B F$, and finally produce a series of physiological and biochemical anti-stress reactions.

Jasmonic acid and its derivatives activate the signaling pathways similar to ABA (Riemann et al., 2015). Studies have suggested that jasmonate ZIM (zinc-finger inflorescence meristem) domain (JAZ) proteins in the JA signaling pathway may be involved in the generation of a response to drought (Liu et al., 2017). JAZ family members act as repressors of JA signals, which directly interact with the basic helix-loop-helix leucine zipper-type (bHLH zip-type) factor MYC2 (Melotto et al., 2008). MYC2, a key transcriptional activator that regulates JAdependent transcriptional reprogramming (Boter et al., 2004). In this pathway, the transcription repressor JAZ protein interacts with the downstream positive regulatory factor $M Y C 2$, so as to inhibit the regulation of MYC2 on downstream gene expression (Hou et al., 2010). When plants are subjected to environmental stresses, the stress signals improve the production of JA in 
BRASSINOSTEROD B IOSYNTHESIS

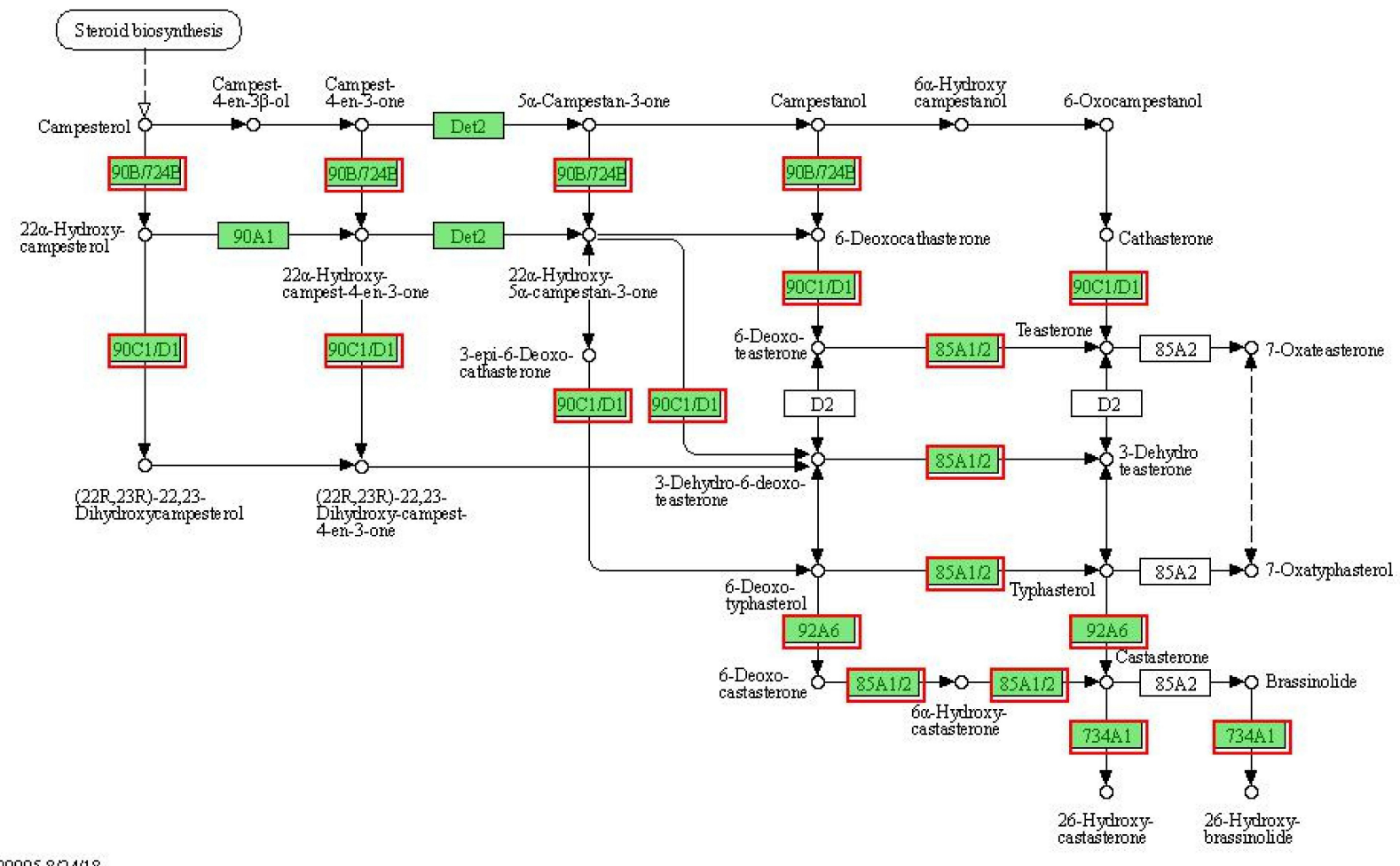

(c) Kanehisa Laboratories

FIGURE 10 | Differentially expressed genes involved the "brassinosteroid biosynthesis" pathway. The red boxes indicated the DEGs were involved in the "brassinosteroid biosynthesis" pathway.

peroxidase, and then the JA is transported to the cytoplasm to form jasmonoyl-isoleucine (JA-Ile) by JA-Ile synthase (JAR1); JAIle promotes the ubiquitin ligase complex ( $\mathrm{SCF}^{\mathrm{Coi} 1}$ ) to form a complex with JAZ, and then $26 \mathrm{~S}$ protease hydrolyzes the JAZ protein, releasing MYC2, which can be combined with the G-box of downstream target genes, thus affecting the expression of downstream genes (Wasternack and Strnad, 2016; Wasternack and Song, 2017). Here, under drought stress conditions, 3 JAR1 genes and $3 J A Z$ genes were down-regulated while $4 M Y C 2$ genes were up-regulated, indicating that the down-regulation of JAR1 and $J A Z$ genes might promote the dissociation and release of MYC2 and then lead to the stress response of MCB.

Auxin is an important regulatory signal in the process of plant growth. It mainly plays a role in the vigorous tissue, but it is also considered to be a negative regulator of plant drought resistance (De and Jürgens, 2007). In the auxin signal transduction pathway, the extracellular receptor senses the changes of the external environment, the signal is transmitted to the cell through a variety of related pathways, then the nuclear auxin receptor receives the signal, and the complex process of gene expression regulation starts (Chen et al., 2007). Plant auxin primary response genes include three main categories: AUX/IAA, SAUR, and GH3 gene families (Liscum and Reed, 2002). Small auxin up-RNA (SAUR) genes promote cell expansion (Chae et al., 2012). SAUR proteins provide a mechanistic link between auxin and plasma membrane $\mathrm{H}^{+}$ATPases in Arabidopsis thaliana and interact with the PP2CD subfamily of type 2C protein phosphatases (Spartz et al., 2014). The plant GH3 gene family is a typical auxin-responsive gene family, which is involved in the auxin signaling pathway, light signaling, and plant defense responses (Li et al., 2008). The promoter of plant $G H 3$ usually contains auxin response element $(A u x R E)$ TGTCTC sequence, which can specifically bind to auxin response factor $(A R F)$ (Ulmasov et al., 1997). Auxin signal transduction is mainly completed through the interaction between $A R F$ and auxin/indole-3-acetic acid (AUX/IAA) (Piya et al., 2014). When the auxin concentration in plants is at aw level, $A U X / I A A$ binds to $A R F$ to prevent $A R F$ from activating auxin-related gene transcription; When auxin concentration increases, $A U X / I A A$ binds to auxin receptor TIR and is degraded, releasing $A R F$ to regulate the expression of auxin responserelated genes (Guilfoyle and Hagen, 2007). In the present study, the $A U X / I A A, A R F$, and SAUR genes responded to drought stress in this pathway. Among them, 6 up-regulation and 1 downregulation genes were involved in AUX/IAA, 1 up-regulation gene was in involved ARF, and 2 up-regulation and 7 downregulation genes were involved in SAUR. It was inferred that the expression of $A U X / I A A$ contributed to the expression and 


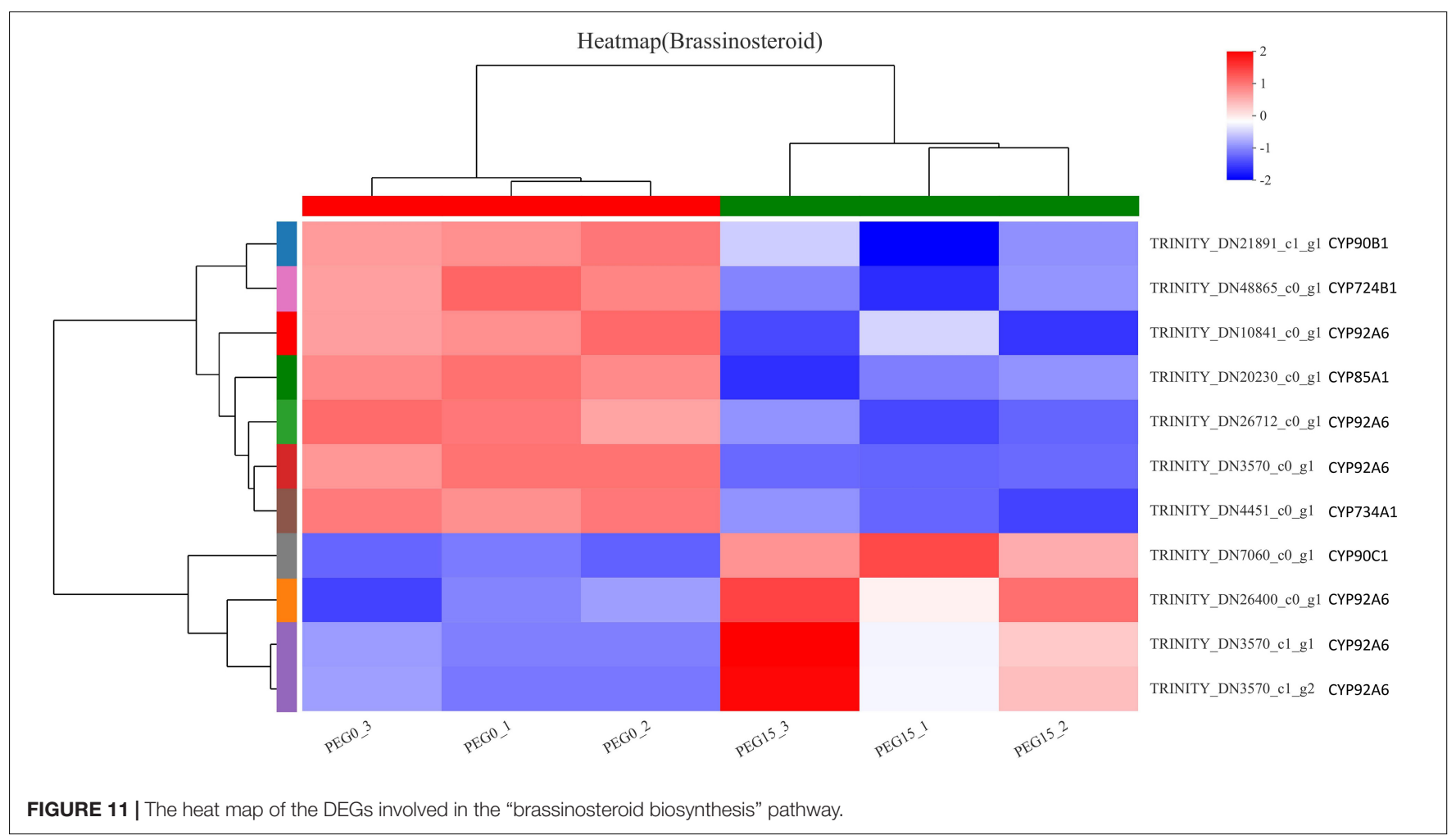

release of ARF protein and the expression of SAUR, thus affecting plant growth of MCB.

Phytohormone ethylene affects a series of developmental processes and stress-resistance responses in plants. In A. thaliana, the ethylene signal transduction pathway has been well studied. Briefly, under the action of $\mathrm{Cu}^{+}$, ethylene molecules bind to ethylene receptors (ETR1, ERS1, ETR2, ERS2, and EIN4), resulting in the inactivation of the receptor CTR1 complex. The inactivated CTR1 no longer phosphorylates the downstream EIN2, consequently, the EIN2 is activated because it is not degraded. Then, the carboxyl end of EIN2 protein is cleaved and dissociated and enters the nucleus (Ji and Guo, 2013). EIN2 center may promote the accumulation of EIN3/EIL1 in the nucleus by inhibiting the ubiquitination and degradation of EIN3/EIL1 mediated by EBF1/2 protein (Guo and Ecker, 2003). EIN3/EIL1 activates downstream target gene expression such as ethylene response factor 1 (ERF1) at the transcriptional level. Meanwhile, ERF1, as a TF also activates the expression of downstream target genes. Subsequently, a large number of downstream ethylene response genes are activated at the transcriptional level, resulting in ethylene reaction (Zhao and Guo, 2011). In our investigation, 2 ETR genes and 1 ERF1 gene were induced to be differentially expressed by drought stress and they were down-regulated compared to the normal conditions. ERFs are unique to plants and belong to the AP2/EREBP-type TFs, which play a role of trans-acting factors at the last step of ethylene signaling (Klee and Giovannoni, 2011; Xiao et al., 2013). ETR is one of the key elements in the ethylene signal transduction pathway, which plays an important role in regulating plant growth and development and resisting stress (Bisson and Groth,
2010). Therefore, it was indicated that ETR and ERF1 might be involved in responses to drought stress of MCB.

Cytokinins play important roles in plant growth and development and stress tolerance (Sakakibara, 2006). Cytokinin signal transduction involves a multistep two-component system consisting of Arabidopsis histidine phosphotransfer proteins (AHP), histidine kinases (AHK), response regulators (ARRs), and so on (Hwang and Sheen, 2001). Cytokinin receptors AHK2, AHK3, and CRE1 bind to cytokinin, self phosphorylate, and transfer the phosphate groups from the histidine residues conserved in kinase region to aspartate residues conserved in signal receiving region; The phosphate groups on aspartic acid are transferred to Arabidopsis histidine-phosphotransfer proteins (AHPs) in the cytoplasm; The phosphorylated AHPs enter the nucleus and transfer the phosphate groups to Arabidopsis response regulators (ARRs), regulating the downstream cytokinin reaction, so as to produce a series of biochemical effects and regulate plant growth and development (Sakakibara, 2006; Muller and Sheen, 2007). In this pathway, our results exhibited 3 DEGs (1 up-regulated and 2 down-regulated), 6 DEGs (5 up-regulated and 1 down-regulated), and 2 DEGs (upregulated) were in AHP, A-ARR, and AHK, respectively. OsAHPs function as positive regulators of the cytokinin signaling pathway and the expression of OsAHP1 and OsAHP2 are inhibited by drought stress (Sun et al., 2014). The mutations in a-arr5, a-arr6, or $a$-arr7 lead to enhanced cold tolerance, suggesting that the $A-A R R 5, A-A R R 6$, and $A-A R R 7$ negatively regulate cold stress responses (Jeon et al., 2010). AHK1 is considered to be a positive regulator of osmotic stress signaling because the overexpression of $A H K 1$ shows enhanced stress tolerance, 


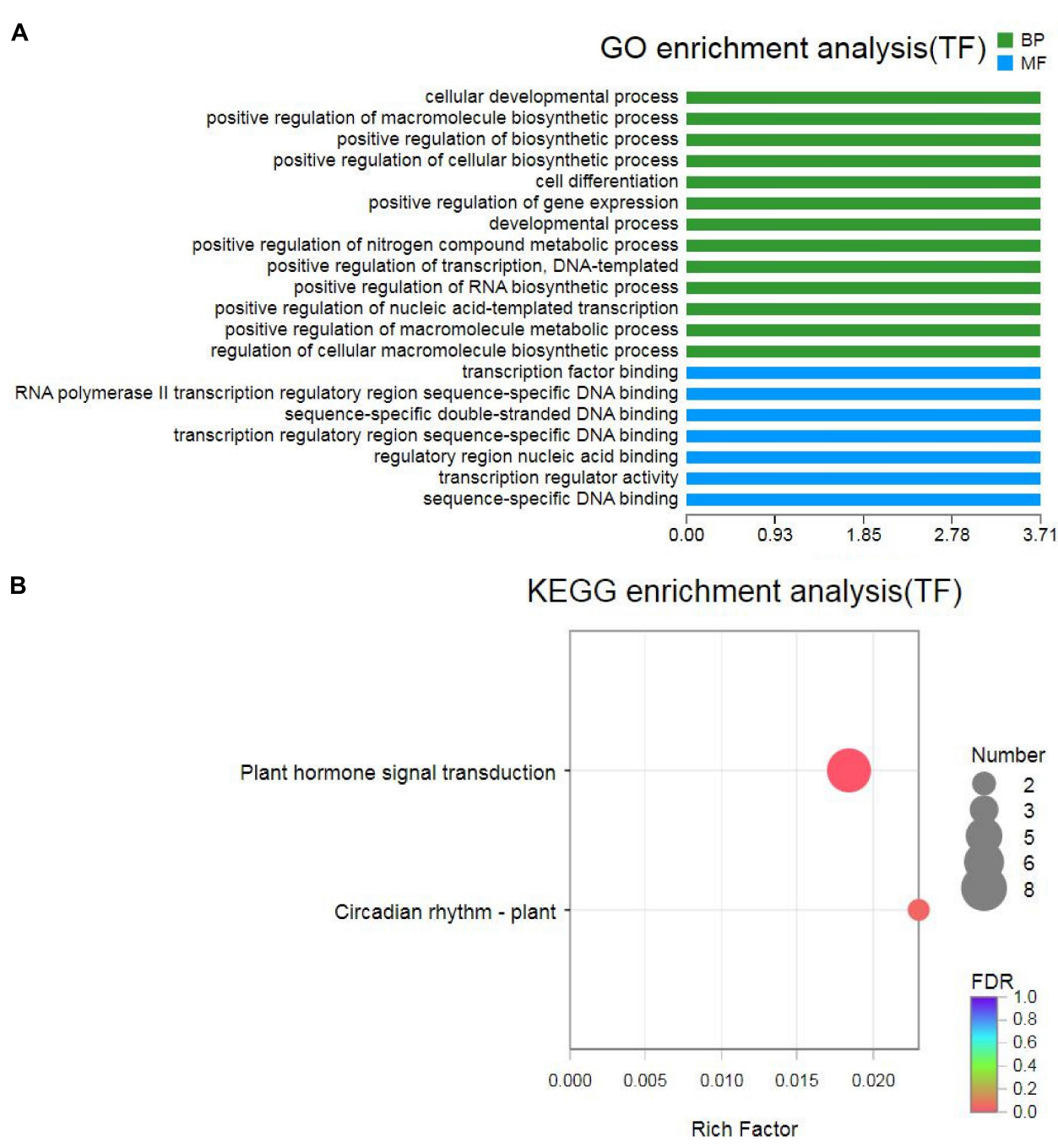

FIGURE 12 | Gene Ontology and KEGG analysis of TFs. (A) GO analysis of TFs; (B) KEGG analysis of TFs.

while ahk1 mutant is sensitive to osmotic stress (Tran et al., 2007; Wohlbach et al., 2008). Thus, it was indicated that the 2 down-regulated $A H P$ genes, 1 down-regulated $A-A R R$ gene, and 2 down-regulated $A H K$ genes might be involved in drought resistance response in $\mathrm{MCB}$.

Brassinolide (BR) is an important steroidal hormone and plays an important role in promoting plant growth and improving plant stress resistance (Hao et al., 2017). Within the BR signal transduction, BR is recognized by membrane receptor BRI1 (Wang et al., 2012), which dissociates its inhibitor, BRI1 associated receptor kinase 1 (BAK1), from the plasma membrane; At the same time, the BRI1-associated receptor kinase 1 (BAK1) on the membrane forms an allotropic polymer with BRI1; The BRI1-BAK1 complex inhibits the activity of downstream brassinosteroid insensitive 2 (BIN2) kinase and activates the activity of BRI1 suppressor 1 (BSU1) phosphatase, which changes the phosphorylation level of TFs BES1 and BZR1; BES1 and BZR1 directly bind to the promoter of BRs related genes and regulate their expression positively or negatively (Belkhadir et al.,
2006). BAK1 (BRI1 associated receptor kinase 1) is a receptor kinase rich in Leu repeats, which can regulate brassinolide receptor BRI1 and make plants resistant to bacterial antigens (Chinchilla et al., 2007). The bak1-bkk1 mutants show plant lethal phenotype, callose deposition, ROS accumulation, and even spontaneous cell death under poor growth conditions, indicating that $\mathrm{BAK} 1$ and $\mathrm{BKK} 1$ positively regulate the plant growth pathway dependent on $\mathrm{BR}$ and negatively regulate the apoptosis pathway independent of BR (He et al., 2007). Brassinosteroid signaling kinase (BSKs) family members can interact with and be phosphorylated by BRI1 (Tang et al., 2008). CYCD is a crucial regulatory factor in the G1 phase and can promote the transformation of cells from the G1 phase to the $S$ phase, which plays an important role in regulating cell division, size, quantity, and organ morphogenesis (Bisbis et al., 2006). TCH4 gene, encoding xyloglucan endoglycosylase, can transfer xyloglucan in the cell wall during plant morphogenesis and affect the formation and degradation of the cell wall, thus playing an important role in plant secondary growth, disease resistance, 
TABLE 2 | qPCR verification.

\begin{tabular}{lccc}
\hline Gene Id & KO name & $\begin{array}{c}\text { RNA-seq (fold } \\
\text { change) }\end{array}$ & $\begin{array}{c}\text { qPCR (fold } \\
\text { change) }\end{array}$ \\
\hline TRINITY_DN26712_C0_g1 & CYP92A6 & 0.07 & 0.46 \\
TRINITY_DN7060_C0_g1 & CYP90C1, ROT3 & 2.09 & 9.52 \\
TRINITY_DN21891_c1_g1 & CYP90B1, DWF4 & 0.49 & 1.13 \\
TRINITY_DN48865_c0_g1 & CYP724B1, D11 & 0.28 & 0.58 \\
TRINITY_DN3570_C1_g1 & CYP92A6 & 150.85 & 13.88 \\
TRINITY_DN26400_C0_g1 & CYP92A6 & 2.73 & 8.96 \\
TRINITY_DN20230_C0_g1 & CYP85A1, BR6OX1 & 0.47 & 1.40 \\
TRINITY_DN4451_C0_g1 & CYP734A1, BAS1 & 0.22 & 1.62 \\
TRINITY_DN3232_C0_g1 & ABF & 0.39 & 0.50 \\
TRINITY_DN4161_C0_g1 & K14486, ARF & 2.43 & 2.76 \\
TRINITY_DN3183_C0_g2 & ABF & 2.14 & 3.53 \\
TRINITY_DN28414_C0_g2 & ERF1 & 0.20 & 0.47 \\
\hline
\end{tabular}

and stress resistance (Xu et al., 1995; Zhang et al., 2008). In our study, the results showed that the BRI (included 1 DEGs, up-regulation), $B K I$ (included 1 DEGs, down-regulation), $B A K$ (included 2 DEGs, down-regulation), BSK (included 1 DEGs, up-regulation), TCH4 (included 1 DEGs, up-regulation), and CYCD3 (included 1 DEGs, up-regulation) were found to be differentially expressed under drought stress conditions. In this pathway, the up-regulated expression of BRI might promote the $B S K$ expression, leading to the up-regulated expression of $\mathrm{TCH} 4$ and $C Y C D 3$, finally resulting in the drought stress responses of MCB. Overall, the genes involved in different steps of the "plant hormone signal transduction" pathway were significantly differential expression under drought stress, indicating that when MCB encountered drought stress, the MCB plants might adapt to drought conditions by regulating the expression of related genes in the "plant hormone signal transduction" pathway.

In addition, in the EHT and JA signaling pathways, ethyleneresponsive element-binding factors (ERFs) form a plant-specific transcriptional factor superfamily of 147 members in A. thaliana (Nakano et al., 2006) and influence a number of developmental processes and are also important for adaptation to biotic or abiotic stresses (Cheng et al., 2013). The expression of multiple members of the APETALA2/ETHYLENE RESPONSE FACTOR (AP2/ERF) family requires the synergistic effect of ETH and JA signals (Zhou et al., 2010). MYC2, as the core TF of the JA signal transduction pathway, is involved in regulating plant growth, development, and stress response. In this study, ERF1 inhibited the production of MYC2 metabolites and MYC2 also inhibited the production of ERF1 metabolites in the "MAPK signaling pathway-plant" pathway (Figure 8B). It was demonstrated that ERF1 was proposed to be an integrator of ETH and JA defense responses (Lorenzo et al., 2003). This suggested that the phytohormones (ETH and JA) might coordinate responses to drought stress in MCB.

\section{Brassinosteroid Biosynthesis Involved in Response to Drought Stress}

As mentioned above, the KEEG results of DEGs revealed that brassinosteroid biosynthesis was also one of the most significant enrichment pathways (Table 1). Therefore, we speculated that the brassinosteroid biosynthesis might play important role in response to drought stress in MCB. Our data showed that 11 unigenes, including CYP90B1, CYP90C1, CYP85A1, CYP724B1, CYP734A1, and CYP92A6 were involved in brassinosteroid biosynthesis. In $A$. thaliana, the transcript levels of CYP90 and CYP85 genes are down-regulated by brassinolide, which is the end product of the brassinosteroid biosynthesis pathway. CYP90C1 and CYP85A1 mRNAs are more abundant in roots, whereas CYP90B1 is ubiquitously expressed (Bancos et al., 2002). CYP85A1 and CYP85A2 are two CYP85A gene members in $A$. thaliana and CYP85A2 mediates the conversion of castasterone to brassinolide and CYP85A1 is essential for the initiation of female gametogenesis in A. thaliana (Pérez-España et al., 2011). CYP724B1 gene plays a role in BR synthesis and may be involved in the supply of 6-deoxotyphasterol and typhasterol in the BR biosynthesis network and is associated with the internode elongation and seed development in rice (Tanabe et al., 2005). CYP734As as a key gene in inactivated brassinosteroids, regulate plant growth and development (Nakamura et al., 2005). CYP734A7 encodes a C-26 hydroxylase of brassinosteroids and is probably involved in brassinosteroid catabolism (Ohnishi et al., 2006). ZmCYP92A1 protein has a close relationship with flavonoids 3-monooxygenase of the CYP75 subfamily and is involved in abiotic stresses defense (Duan and Song, 2018). In the present study, the 11 unigenes were differentially expressed under drought stress. It was inferred that the expression of these genes might be closely related to the brassinosteroid biosynthesis. The differential expression of these genes might lead to the change of brassinolide content, thus affecting the growth and development of MCB.

\section{Transcription Factors Involved in Response to Drought Stress}

In this study, we found that 8 DEGs (TFs) might be involved in response to drought stress in MCB (Supplementary Table 9). These TFs included ABA-responsive element ( $A B R E)$-binding transcription factor1 (AREB1) (TRINITY_DN3232_c0_g1), auxin response factor (ARF9) (TRINITY_DN4161_c0_g1), abscisic acidinsensitive 5-like protein (ABI5) (TRINITY_DN3183_c0_g2), ethylene-responsive transcription factor ERF1b (ERF1b) (TRINITY_DN28414_c0_g2), phytochrome-interacting factors (PIF3) (TRINITY_DN9557_c0_g1), etc. AREB1 regulates ABREdependent $\mathrm{ABA}$ signaling that enhances drought tolerance in vegetative tissues (Fujita et al., 2005). AREB1 is a basic domain/leucine zipper (bZIP) TF that binds to the ABAresponsive element $(\mathrm{ABRE})$ motif in the promoter region of ABA-inducible genes; The bZIP proteins function to activate plant genes responsive to various physiological, developmental, and environmental signals, and the molecular mechanisms that may control gene-specific transcriptional activation involve post-translational modification, heterodimerization, and nuclear localization (Foster et al., 1994). ABI5, also belonging to the 13-member group A of Arabidopsis bZIP TFs (Jakoby et al., 2002) contributes to the induction of desiccation tolerance 


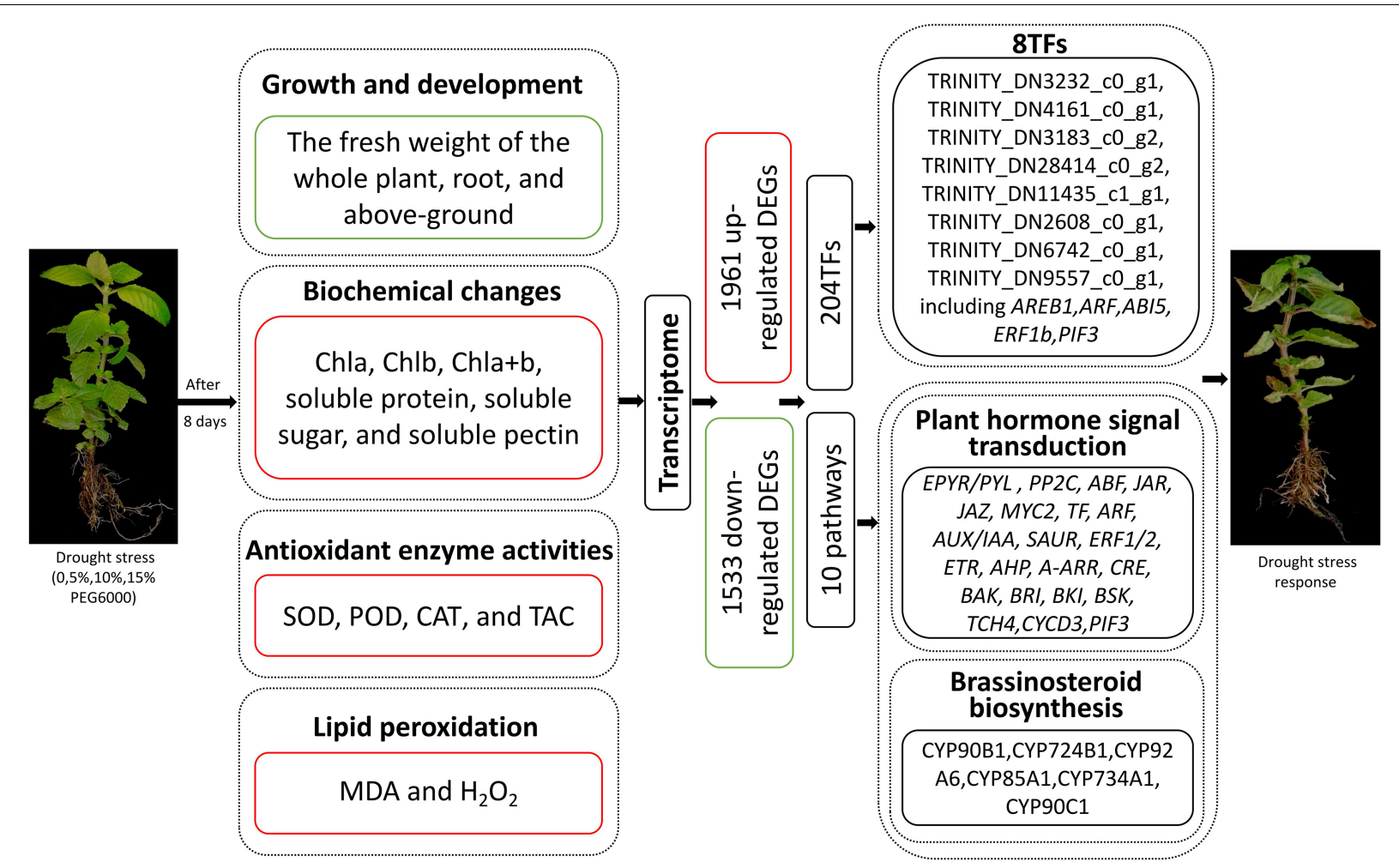

FIGURE 13 | Overview of drought stress affecting the growth and development of MCB. Red boxes and words indicated up-regulation; green boxes indicated down-regulation.

(Maia et al., 2014). Therefore, in this study, the AREB1 and ABI5 might activate ABA-inducible genes through post-translational modification, heterodimerization, or nuclear localization so as to respond to drought stress.

Auxin response factors are involved in plant growth and developmental processes. ARFs are TFs that bind with specificity to TGTCTC auxin response elements (AuxRE) in promoters of the auxin response genes (Guilfoyle and Hagen, 2001). ARFs function in combination with Aux/IAA (auxin/indole acetic acid) repressors, which dimerize with ARF activators in an auxin-regulated manner (Guilfoyle and Hagen, 2007). ARF7 and ARF19 activators regulate the expression of the ARF19 gene and a subset of auxin response genes (Wilmoth et al., 2005). arf7 arf 19 double mutants have suggested that ARF7 plays a major role and ARF19 a lesser role in regulating a variety of auxin response genes in young seedlings (Okushima et al., 2005). In floral tissues, arf6 arf8 double mutants show the reduced expression of SAUR62-SAUR67 and AUX/IAA genes, indicating that ARF6 and ARF8 may activate at least some auxin response genes (Nagpal et al., 2005). The auxin-inducible GH3 genes (GH3.5 and GH3.6), which are thought to be activated by ARF8 and repressed by ARF17, are the candidate targets for ARF8 and ARF17 (Tian et al., 2004; Mallory et al., 2005). Here, the ARF9 was found to be up-regulated under drought stress conditions. Previous evidence has demonstrated that the $A R F$ transcript abundance is regulated by microRNAs at the posttranscriptional level (Rhoades et al., 2002; Zouine et al., 2014; Shen et al., 2015). Transcriptional regulation and post-transcriptional regulation may play an important role in controlling the levels of different ARF proteins in various cells and tissues and the target genes, containing the AuxRE sequences GAGACA or TGTCTC in their promoter regions may be the potential binding sites for the ARF proteins (Guilfoyle and Hagen, 2007).

In $A$. thaliana, the perception of light signals by the phytochrome (phy) family of sensory photoreceptors (phyAphyE) begins an intracellular transduction process that leads to the changes in the expression of nuclear genes that affect plant growth and developmental responses (Rockwell et al., 2006). PIF3, a direct phytochrome reaction partner in the photoreceptor's signaling network, is a G-box binding bHLH protein that interacts preferentially with the active form of phytochrome and is involved in controlling the expression of light-regulated genes such as CCA1 and LHY (Martinez-Garcia et al., 2000). PIF3 is firstly identified in a yeast two-hybrid screen for phyB-interacting proteins and subsequently found to bind conformer-specifically to the Pfr form of both phyA and phyB in a photoreversible fashion (Ni et al., 1999). In the present study, the MCB plantlets were exposed to the red light condition and the PIF3 (TRINITY_DN9557_c0_g1) was up-regulated under drought stress conditions. These indicated that the PIF3 not only responded to the red light but also might be involved in response to drought stress. 
ERF1b acts as a transcriptional activator and binds to the GCCbox pathogenesis-related promoter element, which is involved in the regulation of gene expression during the plant development, and/or mediated by stress factors and by components of stress signal transduction pathways (Onate-Sanchez and Singh, 2002). Here, the ERF1b interacted with MYC2 in the "MAPK signaling pathway-plant" pathway, indicating that ERF1b might be a key integrator of ETH and JA signals in the regulation of ETH/JAdependent defenses (Lorenzo et al., 2003).

\section{CONCLUSION}

Drought stress significantly limited the growth of MCB. The fresh weight of the whole plant, root, and aboveground was significantly decreased, while the contents of Chla, Chlb, Chla + b, soluble protein, soluble sugar, soluble pectin, SOD, CAT, TAC, MDA, and $\mathrm{H}_{2} \mathrm{O}_{2}$ were dramatically increased with the increasing concentrations of PEG6000. The POD activity increased then decreased with the increase of PEG6000 concentration. Transcriptomic analyses revealed a total of 1,961 up-regulated and 1,533 down-regulated unigenes were identified. KEGG enrichment demonstrated that these DEGs were associated with "plant hormone signal transduction," "brassinosteroid biosynthesis," "plant-pathogen interaction," "starch and sucrose metabolism," "phenylpropanoid biosynthesis," and "pentose and glucuronate interconversions," etc. In addition, a total of 204 TFs were identified in DEGs and 8 TFs were significantly enriched in the "plant hormone signal transduction" pathway. These suggested that the genes and TFs might play important roles in response to drought stress in MCB (Figure 13).

\section{REFERENCES}

Apel, K., and Hirt, H. (2004). Reactive oxygen species: metabolism, oxidative stress, and signal transduction. Annu. Rev. Plant Biol. 55, 373-399. doi: 10.1146/ annurev.arplant.55.031903.141701

Bancos, S., Nomura, T., Sato, T., Molnar, G., Bishop, G. J., Koncz, C., et al. (2002). Regulation of transcript levels of the Arabidopsis cytochrome P450 genes involved in brassinosteroid biosynthesis. Plant Physiol. 130, 504-513. doi: 10.1104/pp.005439

Beck, E. H., Fetting, S., Knake, C., Harting, K., and Bhattarai, T. (2007). Specific and unspecific responses of plants to cold and drought stress. J. Biosci. 32, 501-510. doi: 10.1007/s12038-007-0049-5

Belkhadir, Y., Wang, X. I., and Choy, J. (2006). Arabidopsis brassinosteroid signaling pathwey. Sci. STKE 364:cm5.

Bhargava, S., and Sawant, K. (2013). Drought stress adaptation: metabolic adjustment and regulation of gene expression. Plant Breed. 132, 21-32. doi: $10.1111 /$ pbr.12004

Bisbis, B., Delma, F., Joubès, J., Sicard, A., Hernould, M., Inzé, D., et al. (2006). Cyclin-dependent kinase (CDK) inhibitors regulate the CDK-Cyclin complex activities in endoreduplicating cells of developing tomato fruit. J. Biol. Chem. 281, 7374-7383. doi: 10.1074/jbc.M506587200

Bisson, M. M. A., and Groth, G. (2010). New insight in ethylene signaling: autokinase activity of ETR1 modulates the interaction of receptors and EIN2. Mol. Plant 3, 882-889. doi: 10.1093/mp/ssq036

Boter, M., Ruiz-Rivero, O., Abdeen, A., and Prat, S. (2004). Conserved MYC transcription factors play a key role in jasmonate signaling both in tomato and Arabidopsis. Genes Dev. 18, 1577-1591. doi: 10.1101/gad.2 97704

\section{DATA AVAILABILITY STATEMENT}

The original contributions presented in the study are publicly available. This data can be found here: National Center for Biotechnology Information (NCBI) BioProject database under accession number PRJNA777790.

\section{AUTHOR CONTRIBUTIONS}

DT: conceptualization, methodology, investigation, formal analysis, writing-original draft, and writing-reviewing and editing. DT, SQ, FW, and JM: funding acquisition. YiL, KW, YaL, and CQ: methodology and investigation. All authors approved the submitted version.

\section{FUNDING}

This study was supported by Guangxi Innovation-Driven Development Project (GuiKe AA18242040), the Scientific Research Funding Project of Guangxi Botanical Garden of Medicinal Plants (GuiYaoJi202011), National Natural Science Foundation of China (82160723 and 82060689), Natural Science Foundation of Guangxi (2020JJA140312), and the China Agriculture Research System (CARS-21).

\section{SUPPLEMENTARY MATERIAL}

The Supplementary Material for this article can be found online at: https://www.frontiersin.org/articles/10.3389/fpls.2022. 809723/full\#supplementary-material

Bradford, M. M. (1976). A rapid and sensitive method for the quantitation of microgram quantities of protein utilizing the principle of protein-dye binding. Anal. Biochem. 72, 248-254. doi: 10.1006/abio.1976.9999

Bray, E. A. (1993). Molecular responses to water deficit. Plant Physiol. 103, 1035-1040. doi: 10.1104/pp.103.4.1035

Buysse, J., and Merckx, R. (1993). An improved colorimetric method to quantify sugar content of plant tissue. J. Exp. Bot. 44, 1627-1629.

Cao, Y., Luo, Q., Tian, Y., and Meng, F. (2017). Physiological and proteomic analyses of the drought stress response in Amygdalus mira (Koehne) Yü et Lu roots. BMC Plant Biol. 17:53. doi: 10.1186/s12870-017-1000-z

Chae, K., Isaacs, C. G., Reeves, P. H., Maloney, G. S., Muday, G. K., Nagpal, P., et al. (2012). Arabidopsis SMALL AUXIN UP RNA63 promotes hypocotyl and stamen filament elongation. Plant J. 71, 684-697. doi: 10.1111/j.1365-313X. 2012.05024.x

Chen, Q., Wang, R., Bai, R. X., Zhai, R. T., Cui, B. M., Peng, M., et al. (2007). Auxin signaling and its role in cotton fiber development. High Technol. Lett. 17, 104-110.

Cheng, M. C., Liao, P. M., Kuo, W. W., and Lin, T. P. (2013). The Arabidopsis ETHYLENE RESPONSE FACTOR1 regulates abiotic stress-responsive gene expression by binding to different cis-acting elements in response to different stress signals. Plant Physiol. 162, 1566-1582. doi: 10.1104/pp.113.221911

Chinchilla, D., Zipfel, C., and Robatzek, S. (2007). A flagellin-induced complex of the receptor FLS2 and BAK1 initiates plant defence. Nature 448, 497-500. doi: 10.1038/nature05999

Conesa, A., Gotz, S., Garcia-Gomez, J. M., Terol, J., Talon, M., and Robles, M. (2005). Blast2GO: a universal tool for annotation, visualization and analysis in functional genomics research. Bioinformatics 21, 3674-3676. doi: 10.1093/ bioinformatics/bti610 
De, S. I., and Jürgens, G. (2007). Patterning the axis in plants-auxin in control. Curr. Opin. Genet. Dev. 17, 337-343. doi: 10.1016/j.gde.2007.04.012

Duan, F. M., and Song, W. W. (2018). Cloning and expression of phytoecdysone biosynthesis enzyme gene ZmCYP92A1 in Maize. J. Maize Sci. 26, 21-29.

Emmerich, W. E., and Hardegree, S. P. (1990). Polyethylene glycol solution contact effects on seed germination. Agron. J. 82, 1103-1107. doi: 10.1007/BF00384644

Fahad, S., Bajwa, A. A., Nazir, U., Anjum, S. A., Farooq, A., Zohaib, A., et al. (2017). Crop production under drought and heat stress: plant responses and management options. Front. Plant Sci. 8:1147. doi: 10.3389/fpls. 2017.01147

Fathi, A., and Tari, D. B. (2016). Effect of drought stress and its mechanisms in plants. Int. J. Life Sci. 10, 1-6. doi: 10.1007/978-3-319-28899-4_1

Foster, R., Izawa, T., and Chua, N. H. (1994). Plant bZIP proteins gather at ACGT elements. FASEB J. 8, 192-200. doi: 10.1096/fasebj.8.2.8119490

Fujita, Y., Fujita, M., Satoh, R., Maruyama, K., Parvez, M. M., Seki, M., et al. (2005). AREB1 is a transcription activator of novel ABRE-dependent ABA signaling that enhances drought stress tolerance in Arabidopsis. Plant Cell 17, 3470-3488. doi: $10.1105 /$ tpc. 105.035659

Gao, J. F. (2000). Experimental Techniques of Plant Physiology. Xi,an: World Publishing Corporation.

Giannopolitis, C. N., and Ries, S. K. (1977). Superoxide dismutase I. Occurrence in higher plants. Plant Physiol. 59, 309-314. doi: 10.1104/pp.59.2.309

Gill, S. S., and Tuteja, N. (2010). Reactive oxygen species and antioxidant machinery in abiotic stress tolerance in crop plants. Plant Physiol. Biochem. 48, 909-930. doi: 10.1016/j.plaphy.2010.08.016

Goldblith, S. A., and Proctor, B. E. (1950). Photometric determination of catalase activity. J. Biol. Chem. 187, 705-709.

Goto, S., and Kanehisa, M. (2000). KEGG: Kyoto Encyclopedia of Genes and Genomes. Nucleic Acids Res. 28, 27-30.

Grabherr, M. G., Haas, B. J., Yassour, M., Levin, J. Z., Thompson, D. A., and Amit, I. (2011). Full-length transcriptome assembly from RNA-Seq data without a reference genome. Nat. Biotechnol. 29, 644-652. doi: 10.1038/nbt.1883

Guilfoyle, T. G., and Hagen, G. (2007). Auxin response factors. Curr. Opin. Plant Biol. 10, 453-460.

Guilfoyle, T. J., and Hagen, G. (2001). Auxin response factors. J. Plant Growth Regul. 10, 281-291. doi: 10.1007/s003440010026

Guo, H., and Ecker, J. R. (2003). Plant responses to ethylene gas are mediated by SCF (EBF1/EBF2)- dependent proteolysis of EIN3 transcription factor. Cell 115, 667-677. doi: 10.1016/s0092-8674(03)00969-3

Hammad, S. A. R., and Ali, O. A. M. (2014). Physiological and biochemical studies on drought tolerance of wheat plants by application of amino acids and yeast extract. Ann. Agric. Sci. 59, 133-145.

Hao, L., Zhang, Y. S., Duan, L. S., Zhang, M. C., and Li, Z. H. (2017). Cloning, expression and functional analysis of Brassinosteroid receptor gene ( $Z m B R I 1)$ from Zea mays L. Acta Agron. Sin. 43, 1261-1271.

He, F., Sheng, M., and Tang, M. (2017). Effects of Rhizophagus irregularis on photosynthesis and antioxidative enzymatic system in Robinia pseudoacacia L. under drought stress. Front. Plant Sci. 8:183. doi: 10.3389/fpls.2017.00183

He, K., Gou, X., and Yuan, T. (2007). BAK1 and BKK1 regulate brassinosteroiddependent growth and brassinosteroid-independent cell-death pathways. Curr. Biol. 17, 1109-1115. doi: 10.1016/j.cub.2007.05.036

Hemeda, H. M., and Klein, B. P. (1990). Effects of naturally occurring antioxidants on peroxidase activity of vegetable extracts. J. Food Sci. 55, 184-185. doi: 10. 1111/j.1365-2621.1990.tb06048.X

Hou, X., Lee, L. Y., Xia, K., Yan, Y., and Yu, H. (2010). DELLAs modulate jasmonate signaling via competitive binding to JAZs. Dev. Cell 19, 884-894. doi: 10.1016/ j.devcel.2010.10.024

Hu, Y., Wang, B., Hu, T., Chen, H., Li, H., Zhang, W., et al. (2015). Combined action of an antioxidant defence system and osmolytes on drought tolerance and post-drought recovery of Phoebe zhennan S. Lee saplings. Acta Physiol. Plant. 37:84.

Huang, J., Chen, Q. J., Rong, Y. P., Tang, B., Zhu, L. W., Ren, R. R., et al. (2021). Transcriptome analysis revealed gene regulatory network involved in PEGinduced drought stress in Tartary buckwheat (Fagopyrum Tararicum). PeerJ 9:e11136. doi: 10.7717/peerj.11136

Hussain, H. A., Hussain, S., Khaliq, A., Ashraf, U., Anjum, S. A., Men, S., et al. (2018). Chilling and drought stresses in crop plants: implications, cross talk, and potential management opportunities. Front. Plant Sci. 9:393. doi: 10.3389/ fpls.2018.00393
Hwang, I., and Sheen, J. (2001). Two-component circuitry in Arabidopsis cytokinin signal transduction. Nature 413, 383-389. doi: 10.1038/35096500

Jaberiea, H., Momenib, S., and Nabipour, I. (2020). Total antioxidant capacity assessment by a development of an antioxidant assay based on green synthesized $\mathrm{MnO}_{2}$ nanosheets. Microchem. J. 157:104908. doi: 10.1016/j. microc.2020.104908

Jakoby, M., Weisshaar, B., Droge-Laser, W., Vicente-Carbajosa, J., Tiedemann, J., Kroj, T., et al. (2002). bZIP transcription factors in Arabidopsis. Trends Plant Sci. 7, 106-111.

Jaleel, C. A., Manivannan, P., Wahid, A., Farooq, M., Jasimal-Juburi, H., Somasundaram, R., et al. (2009). Drought stress in plants: a review on morphological characteristics and pigments composition. Int. J. Agric.Biol. 11, 100-105.

Jeon, J., Kim, N. Y., Kim, S., Kang, N. Y., Novák, O., Ku, S. J., et al. (2010). A subset of cytokinin two-component signaling system plays a role in cold temperature stress response in Arabidopsis. J. Biol. Chem. 285, 23371-23386. doi: 10.1074/jbc.M109.096644

Ji, Y., and Guo, H. (2013). From endoplasmic reticulum (ER) to nucleus: EIN2 bridges the gap in ethylene signaling. Mol. Plant 6, 11-14. doi: 10.1093/mp/ sss150

Jiang, Y., Macdonald, S. E., and Zwiazak, J. J. (1995). Effects of cold storage and water stress on water relations and gas ex-change of white spruce (Picea glauca) seedlings. Tree Physiol. 15, 267-273. doi: 10.1093/treephys/15.4.267

Joshi-Saha, A., Valon, C., and Leung, J. (2011). Abscisic acid signal off the STARTing block. Mol. Plant 4, 562-580. doi: 10.1093/mp/ssr055

Kamble, P., Giri, S. P., Mane, R. S., and Tiwana, A. (2015). Estimation of chlorophyll content in young and adult leaves of some selected plants. Univers. J. Environ. Res. Technol. 5, 306-310.

Kang, J. M., Yang, Q. C., and Fan, F. C. (2005). Effects of drought stress on induced protein in the different drought resistance Alfalfa leaf. Acta Agrestia Sin. 13, 199-202.

Kashif, M. (2011). Performance of wheat genotypes under osmotic stress at germination and early seedling growth stage. Afr. J. Biotechnol. 6, 971-975.

Khan, R., Ma, X. H., Shah, S., Wu, X. Y., Shaheen, A., Xiao, L. X., et al. (2020). Drought-hardening improves drought tolerance in Nicotiana tabacum at physiological, biochemical, and molecular levels. BMC Plant Biol. 20:486. doi: 10.1186/s12870-020-02688-7

Khan, R., Zhou, P. L., Ma, X. H., Zhou, L., Wu, Y. H., Ullah, Z., et al. (2019). Transcriptome profiling, biochemical and physiological analyses provide new insights towards drought tolerance in Nicotiana tabacum L. Genes 10:1041. doi: $10.3390 /$ genes 10121041

Klee, H. J., and Giovannoni, J. J. (2011). Genetics and control of tomato fruit ripening and quality attributes. Annu. Rev. Genet. 45, 41-59. doi: 10.1146/ annurev-genet-110410-132507

Kramer, G. F., Norman, H. A., Krizek, D. T., and Mirecki, R. M. (1991). Influence of UV-B radiation on polyamines, lipid peroxidation and membrane lipids in cucumber. Phytochemistry 30, 2101-2108. doi: 10.1016/0031-9422(91)83595-c

Lee, S. C., and Luan, S. (2012). ABA signal transduction at the crossroad of biotic and abiotic stress responses. Plant Cell Environ. 35, 53-60. doi: 10.1111/j.13653040.2011.02426.x

Li, B., and Dewey, C. N. (2011). RSEM: accurate transcript quantification from RNA-Seq data with or without a reference genome. BMC Bioinformatics 12:323. doi: 10.1186/1471-2105-12-323

Li, D. Y., Lu, G., Wang, D. D., and Wang, M. (2010). The influence of Xiancao hypolipidemic tea on the TC and TG metabolism of the experimental rabbits. Chinese Gen. Pract. 13, 9-10.

Li, H., Yao, W., Fu, Y., Li, S., and Guo, Q. (2015). De novo assembly and discovery of genes that are involved in drought tolerance in Tibetan Sophora moorcroftiana. PLoS One 10:e111054. doi: 10.1371/journal.pone.0111054

Li, L. H., and Yi, H. L. (2012). Effect of sulfur dioxide on ROS production, gene expression and antioxidant enzyme activity in Arabidopsis plants. Plant Physiol. Biochem. 58, 46-53. doi: 10.1016/j.plaphy.2012.06.009

Li, Y., Zuo, K. J., and Tang, K. X. (2008). A survey of functional studies of the GH3 gene family in plants. Chin. Bull. Bot. 25, 507-515.

Li, Z., Peng, Y., Pan, M. H., Ma, H. P., and Wang, X. J. (2014). Effects of spermidine on the accumulation of osmoregulation matter in leaves of white clover under PEG stress. Chin. J. Grassland 36, 31-36.

Liang, T. S., Zhao, X. Q., and Gao, J. P. (2021). Effect of EBR on seed germination, seedling growth and key enzyme activities for flavonoid biosynthesis in 
Astragalus membranaceus var. Mongholicus under PEG-induced drought stress. Chin. J. Exp. Tradit. Med. Form. 27, 139-145.

Lin, L. S., Zhang, S., Chan, Y. L., Wang, Z. K., and Lin, H. T. (2013). A review of chemical constituents and medicinal function of Mesona chinensis Benth. Curr. Biotechnol. 3, 448-452.

Liscum, E., and Reed, J. W. (2002). Genetics of Aux/IAA and ARF action in plant growth and development. Plant Mol. Biol. 49, 387-400.

Liu, F., Sun, T. T., Wang, L., Su, W. H., Gao, S. W., Su, Y. C., et al. (2017). Plant jasmonate ZIM domain genes: shedding light on structure and expression patterns of JAZ gene family in sugarcane. BMC Genomics 18:771. doi: 10.1186/ s12864-017-4142-3

Liu, F. L., and Feng, C. L. (2008). In vitro antibacterial test of Hsian-tsao (Mesona chinensis Benth) against avian Escherichia coli. Guangdong J. Anim. Vet. Sci. 33, $17-43$.

Lorenzo, O., Piqueras, R., Sánchez-Serrano, J. J., and Solano, R. (2003). ETHYLENE RESPONSE FACTOR1 integrates signals from ethylene and jasmonate pathways in plant defense. Plant Cell 15, 165-178. doi: 10.1105/tpc. 007468

Love, M. I., Huber, W., and Anders, S. (2014). Moderated estimation of fold change and dispersion for RNA-seq data with DESeq2. Genome Biol. 15:550.

$\mathrm{Lu}, \mathrm{Q}$. X. (2020). Effects of drought stress on resistance physiological and biochemical indexes of Taraxacum mongolicum Hand-Mazz. Hebei: Hebei University of Engineering.

Ma, F. J., Li, D. D., Cai, J., Jiang, D., Cao, W. X., and Dai, T. B. (2012). Responses of wheat seedlings root growth and leaf photosynthesis to drought stress. Chin. J. Appl. Ecol. 23, 724-730.

Maia, J., Dekkers, B. J., Dolle, M. J., Ligterink, W., and Hilhorst, H. W. (2014). Abscisic acid (ABA) sensitivity regulates desiccation tolerance in germinated Arabidopsis seeds. New Phytol. 203, 81-93. doi: 10.1111/nph.12785

Mallory, A. C., Bartel, D. P., and Bartel, B. (2005). MicroRNA-directed regulation of Arabidopsis AUXIN RESPONSE FACTOR17 is essential for proper development and modulates expression of early auxin response genes. Plant Cell 17, 1360-1375. doi: 10.1105/tpc.105.031716

Martinez-Garcia, J., Huq, E., and Quail, P. H. (2000). Direct targeting of light signals to a promoter element-bound transcription factor. Science 288, 859-863. doi: 10.1126/science.288.5467.859

Melotto, M., Mecey, C., Niu, Y., Chung, H. S., Katsir, L., and Yao, J. (2008). A critical role of two positively charged amino acids in the Jas motif of Arabidopsis JAZ proteins in mediating coronatine- and jasmonoyl isoleucine-dependent interactions with the COI1 F-box protein. Plant J. 55, 979-988. doi: 10.1111/ j.1365-313X.2008.03566.x

Michael, I. L., Wolfgang, H., and Simon, A. (2014). Moderated estimation of fold change and dispersion for RNA-seq data with DESeq2. Genome Biol. 15:550. doi: 10.1186/s13059-014-0550-8

Mishra, A. K., and Singh, V. P. (2010). A review of drought concepts. J. Hydrol. 391, 202-216.

Muller, B., and Sheen, J. (2007). Arabidopsis cytokinin signaling pathway. Sci. STKE 407:cm5.

Mun, B. G., Lee, S. U., Park, E. J., Kim, H. H., and Hussain, A. (2017). Analysis of transcription factors among differentially expressed genes induced by drought stress in Populus davidiana. 3 Biotech 7:209. doi: 10.1007/s13205-017-0858-7

Nagpal, P., Ellis, C. M., Weber, H., Ploense, S., Barkawi, L. S., Guilfoyle, T. J., et al. (2005). Auxin response factors ARF6 and ARF8 promote jasmonic acid production and flower maturation. Development 132, 4107-4118. doi: 10.1242/ dev.01955

Nakamura, M., Satoh, T., Tanaka, S., Mochizuki, N., Yokota, T., and Nagatani, A. (2005). Activation of the cytochrome P450 gene, CYP72C1, reduces the levels of active brassinosteroids in vivo. J. Exp. Bot. 56, 833-840. doi: 10.1093/jxb/eri073

Nakano, T., Suzuki, K., Fujimura, T., and Shinshi, H. (2006). Genome-wide analysis of the ERF gene family in Arabidopsis and rice. Plant Physiol. 140, 411-432.

Nakashima, K., Ito, Y., and Yamaguchi-Shinozaki, K. (2009). Transcriptional regulatory networks in response to abiotic stresses in arabidopsis and grasses. Plant Physiol. 149, 88-95. doi: 10.1104/pp.108.129791

Ni, M., Tepperman, J. M., and Quail, P. H. (1999). Binding of phytochrome B to its nuclear signalling partner PIF3 is reversibly induced by light. Nature 400, 781-784. doi: $10.1038 / 23500$

Ohnishi, T., Nomura, T., Watanabe, B., Ohta, D., Yokota, T., Miyagawa, H., et al. (2006). Tomato cytochrome P450 CYP734A7 functions in brassinosteroid catabolism. Phytochemistry 67, 1895-1906. doi: 10.1016/j.phytochem.2006.05. 042

Okushima, Y., Overvoorde, P. J., Arima, K., Alonso, J. M., Chan, A., Chang, C., et al. (2005). Functional genomic analysis of the AUXIN RESPONSE FACTOR gene family members in Arabidopsis thaliana: unique and overlapping functions of ARF7 and ARF19. Plant Cell 17, 444-463. doi: 10.1105/tpc.104.028316

Onate-Sanchez, L., and Singh, K. B. (2002). Identification of Arabidopsis ethyleneresponsive element binding factors with distinct induction kinetics after pathogen infection. Plant Physiol. 128, 1313-1322. doi: 10.1104/pp.010862

Pérez-España, V. H., Sánchez-León, N., and Vielle-Calzada, J. P. (2011). CYP85A1 is required for the initiation of female gametogenesis in Arabidopsis thaliana. Plant Signal. Behav. 6, 321-326. doi: 10.4161/psb.6.3.13206

Piya, S., Shrestha, S. K., Binder, B., Stewart, C. N., and Hewezi, T. (2014). Proteinprotein interaction and gene co-expression maps of ARFs and Aux/IAAs in Arabidopsis. Front. Plant Sci. 5:744. doi: 10.3389/fpls.2014.00744

Ren, Q. W., Li, X. H., Yin, X. L., and Li, J. J. (2017). Drought disaster identification and changing tendency in Guangdong province. Trop. Geogr. 37, 494-500.

Rhoades, M. W., Reinhart, B. J., Lim, L. P., Burge, C. B., Bartel, B., and Bartel, D. P. (2002). Prediction of plant microRNA targets. Cell 110, 513-520.

Riemann, M., Dhakarey, R., Hazman, M., Miro, B., Kohli, A., and Nick, P. (2015). Exploring jasmonates in the hormonal network of drought and salinity responses. Front. Plant Sci. 6:1077. doi: 10.3389/fpls.2015.01077

Rockwell, N. C., Su, Y. S., and Lagarias, J. C. (2006). Phytochrome structure and signaling mechanisms. Annu. Rev. Plant Biol. 57, 837-858. doi: 10.1146/ annurev.arplant.56.032604.144208

Rubinstein, B., and Turner, N. C. (1982). Regulation of $\mathrm{H}^{+}$excreation: effects of osmotic shock. Plant Physiol. 69, 939-944. doi: 10.1104/pp.69.4.939

Ryu, H., and Cho, Y. G. (2015). Plant hormones in salt stress tolerance. J. Plant Biol. 58, 147-155. doi: 10.1007/s12374-015-0103-z

Sakakibara, H. (2006). Cytokinins: activity, biosynthesis, and translocation. Аnnu. Rev. Plant Biol. 57, 431-449. doi: 10.1146/annurev.arplant.57.032905.105231

Sharma, A., Ramakrishnan, M., Khanna, K., Landi, M., Prasad, R., Bhardwaj, R., et al. (2022). Brassinosteroids and metalloids: regulation of plant biology. J. Hazard. Mater. 424:127518. doi: 10.1016/j.jhazmat.2021.127518

Sharma, P., Jha, A. B., Dubey, R. S., and Pessarakli, M. (2012). Reactive oxygen species, oxidative damage, and antioxidative defense mechanism in plants under stressful conditions. J. Bot. 2012:217037.

Shen, C. J., Yue, R. Q., Sun, T., Zhang, L., Xu, L. Q., Tie, S. G., et al. (2015). Genomewide identification and expression analysis of auxin response factor gene family in Medicago truncatula. Front. Plant Sci. 6:73. doi: 10.3389/fpls.2015.00073

Shtereva, L., Stoimenova, E., Drumeva-Yoncheva, M., Michailova, B., Kartzeva, T., and Ivanova, V. R. (2017). Response of virginia (flue-cured) tobacco genotypes to water-deficit stress. Arch. Biol. Sci. 69, 119-127. doi: 10.2298/abs160202085s

Silva, E. N., Ferreira, S. L., Viegas, R. A., and Silveira, J. A. G. (2010). The role of organic and inorganic solutes in the osmotic adjustment of drought-stressed Jatropha curcas plants. Environ. Exp. Bot. 69, 279-285. doi: 10.1016/j.envexpbot. 2010.05.001

Spartz, A. K., Ren, H., Park, M. Y., Grandt, K. N., Lee, S. H., Murphy, A. S., et al. (2014). SAUR inhibition of PP2C-D phosphatases activates plasma membrane $\mathrm{H}^{+}$-ATPases to promote cell expansion in Arabidopsis. Plant Cell 26, 21292142. doi: $10.1105 /$ tpc.114.126037

$\mathrm{Su}, \mathrm{H}$. L., Li, S., and Chen, J. Y. (2008). Research progress of Mesona chinensis Benth. Res. Pract. Chin. Med. 22, 79-81.

Sun, L. J., Zhang, Q., Wu, J. X., Zhang, L. Q., Jiao, X. W., Zhang, S. G., et al. (2014). Two rice authentic histidine phosphotransfer proteins, OsAHP1 and OsAHP2, mediate cytokinin signaling and stress responses in rice. Plant Physiol. 165, 335-345. doi: 10.1104/pp.113.232629

Suzuki, N. (2016). Hormone signaling pathways under stress combinations. Plant Signal. Behav. 11:e1247139. doi: 10.1080/15592324.2016.1247139

Tanabe, S., Ashikari, M., Fujioka, S., Takatsuto, S., Yoshida, S., Yano, M., et al. (2005). A novel cytochrome 450 is implicated in brassinosteroid biosynthesis via the characterization of a rice dwarf mutant, dwarf11, with reduced seed length. Plant Cell 17, 776-790. doi: 10.1105/tpc.104.024950

Tang, D. F., Huang, Q. F., Wei, K. H., Yang, X. N., Wei, F., and Miao, J. H. (2021). Identification of differentially expressed genes and pathways involved in growth and development of Mesona chinensis Benth under red and blue light conditions. Front. Plant Sci. 12:761068. doi: 10.3389/fpls.2021.7 61068 
Tang, D. F., Wei, F., Cai, Z. Q., Wei, Y. Y., Aziz, K., Miao, J. H., et al. (2020). Analysis of codon usage bias and evolution in the chloroplast genome of Mesona chinensis Benth. Dev. Genes Evol. 231, 1-9. doi: 10.1007/s00427-020-00670-9

Tang, D. F., Wei, F., Qin, S. X., Aziz, K., Muhammad, H. K., and Zhou, R. Y. (2019). Polyethylene glycol induced drought stress strongly influences seed germination, root morphology and cytoplasm of different kenaf genotypes. Ind Crop. Prod. 137, 180-186. doi: 10.1016/j.indcrop.2019.01.019

Tang, W., Kim, T., Osesprieto, J. A., Sun, Y., Deng, Z., Zhu, S., et al. (2008). BSKs mediate signal transduction from the receptor kinase BRI1 in Arabidopsis. Science 321, 557-560. doi: 10.1126/science.1156973

Tarkow, H., Feist, W. C., and Southerland, C. F. (1996). Interaction of wood and polymeric materials. Penetration versus molecular size. For. Prod. J. 16, 61-65.

Tian, C. E., Muto, H., Higuchi, K., Matamura, T., Tatematsu, K., Koshiba, T., et al. (2004). Disruption and overexpression of auxin response factor 8 gene of Arabidopsis affect hypocotyl elongation and root growth habit, indicating its possible involvement in auxin homeostasis in light condition. Plant J. 40, 333-343. doi: 10.1111/j.1365-313X.2004.02220.x

Tran, L. S., Urao, T., Qin, F., Maruyama, K., Kakimoto, T., Shinozaki, K., et al. (2007). Functional analysis of AHK1/ATHK1 and cytokinin receptor histidine kinases in response to abscisic acid, drought, and salt stress in Arabidopsis. Proc. Natl. Acad. Sci. U. S. A. 104, 20623-20628. doi: 10.1073/pnas.070654 7105

Turkan, I., Bor, M., Ozdemir, F., and Koca, H. (2005). Differential responses of lipid peroxidation and antioxidants in the leaves of drought-tolerant $P$. acutifolius Gray and drought-sensitive $P$. vulgaris L. subjected to polyethylene glycol mediated water stress. Plant Sci. 168, 223-231.

Ulmasov, T., Hagen, G., and Guilfoyle, T. J. (1997). ARF1, a transcription factor that binds to auxin response elements. Science 276, 1865-1868. doi: 10.1126/ science.276.5320.1865

Velikova, V., Yordanov, I., and Edreva, A. (2000). Oxidative stress and some antioxidant systems in acid rain-treated bean plants: protective role of exogenous polyamines. Plant Sci. 151, 59-66.

Wang, R., Li, X. G., Li, S. P., Wang, L. X., and Huang, M. J. (2010). Changes of drought stress on main osmotic adjustment substance in leaves and roots of two banana plantlets. Genomics Appl. Biol. 29:518522.

Wang, Z. Y., Bai, M. Y., Oh, E., and Zhu, J. Y. (2012). Brassinosteroid signaling network and regulation of photomorphogenesis. Annu. Rev. Genet. 46, 701724. doi: 10.1146/annurev-genet-102209-163450

Wasternack, C., and Song, S. H. (2017). Jasmonates: biosynthesis, metabolism, and signaling by proteins activating and repressing transciption. J. Exp. Bot. 68, 1303-1321. doi: 10.1093/jxb/erw443

Wasternack, C., and Strnad, M. (2016). Jasmonate signaling in plant stress responses and development- active and inactive compounds. New Biotechnol. 33, 604-613. doi: 10.1016/j.nbt.2015.11.001

Wilmoth, J. C., Wang, S., Tiwari, S. B., Joshi, A. D., Hagen, G., Guilfoyle, T. J., et al. (2005). NPH4/ARF7 and ARF19 promote leaf expansion and auxin-induced lateral root formation. Plant J. 43, 118-130. doi: 10.1111/j.1365-313X.2005. 02432.x

Wohlbach, D. J., Quirino, B. F., and Sussman, M. R. (2008). Analysis of the Arabidopsis histidine kinase ATHK1 reveals a connection between vegetative osmotic stress sensing and seed maturation. Plant Cell 20, 1101-1117. doi: 10.1105/tpc.107.055871

Wu, H., Wu, X., Li, Z., Duan, L. S., and Zhang, M. (2012). Physiological evaluation of drought stress tolerance and recovery in cauliflower (Brassica oleracea L.) seedlings treated with methyl jasmonate and coronatine. J. Plant Growth Regul. 31, 113-123.

Xiao, Y. Y., Chen, J. Y., Kuang, J. F., Shan, W., Xie, H., Jiang, Y. M., et al. (2013). Banana ethylene response factors are involved in fruit ripening through their interactions with ethylene biosynthesis genes. J. Exp. Bot. 64, 2499-2510. doi: 10.1093/jxb/ert108

Xie, C., Mao, X., Huang, J., Ding, Y., Wu, J., and Dong, S. (2011). KOBAS 2.0: a web server for annotation and identification of enriched pathways and diseases. Nucleic Acids Res. 39, W316-W322. doi: 10.1093/nar/gkr483

$\mathrm{Xu}$, W., Cui, K. H., and Xu, A. H. (2015). Drought stress condition increases root to shoot ratio via alteration of carbohydrate partitioning and enzymatic activity in rice seedlings. Acta Physiol. Plant. 37:9.

Xu, W., Purugganan, M. M., Polisensky, H. D., Antosiewicz, D. M., Fry, S. C., and Braam, J. (1995). Arabidopsis TCH4, regulated by hormones and the environment, encodes a Xyloglucan endotransglycosylase. Plant Cell 7, 15551567. doi: 10.1105/tpc.7.10.1555

Yan, F., Zhu, Y., Zhao, Y., Wang, Y., Li, J., Wang, Q., et al. (2020). De novo transcriptome sequencing and analysis of salt-, alkali-, and drought-responsive genes in Sophora alopecuroides. BMC Genomics 21:423. doi: 10.1186/s12864020-06823-4

Yan, H. X., Fang, L. B., and Huang, D. Z. (2011). Effects of drought stress on the biomass distribution and photosynthetic characteristics of cluster mulberry. Chin. J. Appl. Ecol. 12, 3365-3370.

Yancey, P. H. (2001). Water stress, osmolytes and proteins. Am. Zool. 41, 699-709.

Yang, S. L., Chen, K., Wang, S. S., and Gong, M. (2015). Osmoregulation as a key factor in drought hardening-induced drought tolerance in Jatropha curcas. Biol. Plant 59, 529-536.

Yeha, C. T., Huang, W. H., and Yen, G. C. (2009). Antihypertensive effects of Hsian-tsao and its active compound in spontaneously hyperten-sive rats. J. Nutr. Biochem. 20, 866-875. doi: 10.1016/j.jnutbio.2008.07.015

Yen, G. C., and Hung, C. Y. (2000). Effects of alkaline and heat treatment on antioxidative activity and total phenolics of extract from Hsian-tsao (Mesona procumbens Hemsl). Food Res. Int. 33, 487-492.

Yonny, M. E., Torressi, A. R., Nazareno, M. A., and Cerutti, S. (2017). Development of a novel, sensitive, selective, and fast methodology to determine malondialdehyde in leaves of melon plants by ultra-high-performance liquid chromatographytandem mass spectrometry. J. Anal. Methods Chem. 2017:4327954. doi: 10.1155/2017/4327954

Zhang, C. Q., Wang, J., and Gao, X. (2008). Computational identification of transcriptional regulatory elements in Arabidopsis TCH4 promoter. Hereditas 30, 620-626. doi: 10.3724/sp.j.1005.2008.00620

Zhang, J., Jia, W., Yang, J., and Ismail, A. M. (2006). Role of ABA in integrating plant responses to drought and salt stresses. Field Crop. Res. 97, 111-119.

Zhao, Q., and Guo, H. W. (2011). Paradigms and paradox in the ethylene signaling pathway and interaction network. Mol. Plant 4, 626-634. doi: 10.1093/mp/ ssr042

Zhao, Z. G., Shi, Y. P., Huang, N. Z., Fu, C. M., Tang, F. L., and Jiang, Q. Y. (2011). The research advances on Mesona chinensis Benth in China. J. South. Agric. 42, 657-660.

Zhong, L., Liao, P. R., Liu, C. Z., Qian, J. P., He, W. C., Luo, B., et al. (2021). Effects of drought stress on physiological and biochemical and chemical components of Cinnamomum cassia seedlings. Chin. J. Chin. Mater. Med. 46, 2158-2166. doi: 10.19540/j.cnki.cjcmm.20210224.101

Zhou, M. L., Hou, H. L., Zhu, X. M., Shao, J. R., Wu, Y. M., and Tang, Y. X. (2010). Molecular regulation of terpenoid indole alkaloids pathway in the medicinal plant, Catharanthus roseus. J. Med. Plants Res. 4, 2760-2772.

Zhou, Y. Y., Zhang, Y., Wang, X. W., Han, X., An, Y., Lin, S. W., et al. (2020). Root-specific NF-Y family transcription factor, $P d N F-Y B 21$, positively regulates root growth and drought resistance by abscisic acid-mediated indoylacetic acid transport in Populus. New Phytol. 227, 407-426. doi: 10.1111/nph.16524

Zouine, M., Fu, Y., Chateigner-Boutin, A. L., Mila, I., Frasse, P., Wang, H., et al. (2014). Characterization of the tomato ARF gene family uncovers a multilevels post-transcriptional regulation including alternative splicing. PLoS One 9:e84203. doi: 10.1371/journal.pone.0084203

Conflict of Interest: The authors declare that the research was conducted in the absence of any commercial or financial relationships that could be construed as a potential conflict of interest.

Publisher's Note: All claims expressed in this article are solely those of the authors and do not necessarily represent those of their affiliated organizations, or those of the publisher, the editors and the reviewers. Any product that may be evaluated in this article, or claim that may be made by its manufacturer, is not guaranteed or endorsed by the publisher.

Copyright (C) 2022 Tang, Quan, Lin, Wei, Qin, Liang, Wei and Miao. This is an open-access article distributed under the terms of the Creative Commons Attribution License (CC BY). The use, distribution or reproduction in other forums is permitted, provided the original author(s) and the copyright owner(s) are credited and that the original publication in this journal is cited, in accordance with accepted academic practice. No use, distribution or reproduction is permitted which does not comply with these terms. 